\title{
A novel structural mechanism of ribosomal stop codon readthrough in VEGF-A
}

\author{
Nico 0. Wagner \\ Harvard University \\ Jerome M. Edwards \\ Harvard University \\ Juliana Abramovich \\ Harvard University

\section{Paromita Gupta} \\ Whitehead Institute for Biomedical Research \\ Harish Swaminathan \\ Whitehead Institute for Biomedical Research \\ Silvi Rouskin \\ Whitehead Institute for Biomedical Research \\ Victoria M. D'Souza ( $\nabla$ dsouza@g.harvard.edu ) \\ Harvard University
}

\section{Research Article}

Keywords: VEGF, recoding, translation, NMR, structural biology, RNA

Posted Date: February 9th, 2021

DOI: https://doi.org/10.21203/rs.3.rs-186157/v1

License: (c) (i) This work is licensed under a Creative Commons Attribution 4.0 International License.

Read Full License 
1 A novel structural mechanism of ribosomal stop codon readthrough in 2 VEGF-A

3 Nico O. Wagner ${ }^{1}$, Jerome M. Edwards ${ }^{1}$, Juliana Abramovich ${ }^{1}$, Paromita Gupta ${ }^{2}$, Harish 4 Swaminathan ${ }^{2}$, Silvi Rouskin ${ }^{2}$ and Victoria M. D'Souza ${ }^{1, *}$

5 'Department of Molecular and Cellular Biology, Harvard University, Cambridge, MA. 02138, 6 USA.

$7 \quad{ }^{2}$ Whitehead Institute for Biomedical Research, Cambridge, MA. 02142, USA.

$8 \quad$ "Correspondence: dsouza@g.harvard.edu

10 Abstract

11 The process of ribosomal recoding is generally regulated by an autonomous mRNA signal 12 downstream of stop-codons. While structural studies have provided mechanistic insights into 13 viral systems, no such studies exist in mammalian systems. Here we define a novel structural 14 mechanism for the VEGF-A readthrough system and show that regulation is multifaceted and 15 complex, requiring a multipartite set of RNA elements located at long distances that interact 16 with each other and with hnRNP A2/B1 to synergistically enhance readthrough levels. The Ax17 element downstream of the stop codon adopts a unique multistem (SL-Ax-3) architecture: SL18 Ax 1 interacts with hnRNP A2/B1, while SL-Ax 2 interacts with an RNA element (SL-Au1) located $19 \sim 500 \mathrm{nt}$ upstream at the start of the coding sequence. SL-Au $\mathrm{u}_{1}$ also independently binds to hnRNP A2/B1, which manipulates an equilibrium between alternate structures - from a sequestered bulge towards one that allows for the long-range interaction with SL-Ax2. Overall, our study not only highlights the significance of structural organization of elements within the coding sequence of mRNA, but also provides a functional relevance of the closed-loop mRNA organization in non-canonical translation and suggests complex mechanisms allow for

25 finer integration of many signals for a required output.

\section{Introduction}

Recoding during protein translation, either via programmed ribosomal frameshifting

(PRF) or stop-codon readthrough (PRT), allows for production of extended proteins or polyproteins by bypassing stop codons. While these mechanisms are widely used by RNA viruses 
31 to densely pack information into their small genomes and to maintain relative protein levels ${ }^{1}$,

32 they have also been discovered in cellular systems, including bacteria, yeast, Drosophilae, and

33 humans ${ }^{1,2}$, to regulate a wide range of functions from transcriptional regulation to signal

34 transduction and sub-cellular localization ${ }^{3-5}$. In viruses, ribosomal recoding is essential for

35 maintaining critical relative ratios of proteins, for example structural (Gag) and enzymatic (Pol)

36 in retroviruses and immunomodulatory proteins (in ORF 1a) and replicative proteins (in ORF 1b)

37 in coronaviruses ${ }^{6,7}$. Similarly, bacterial release factor 2 expression levels are auto-regulated via

38 recoding and in Drosophila, recoding of the headcase protein is critical for tracheal

39 development ${ }^{8,9}$.

Studies in viruses have provided some insights into structural elements that drive

42 recoding. However, no such insights currently exist for eukaryotic recoding systems, even though

43 over 300 genes in Drosophila and 100 genes in humans have been identified to potentially 44 undergo functional recoding ${ }^{1,10}$. Very recently, a new isoform of the mammalian vascular

45 endothelial growth factor A (VEGF-A), produced via readthrough, was implicated in regulating 46 levels of angiogenesis ${ }^{11,12}$, indicating that recoding may play a role in regulating critical cellular 47 processes, highlighting the need to understand the underlying mechanisms.

Canonical protein translation requires a complex interplay between ribosomes, mRNA 50 and regulatory protein factors ${ }^{13}$ but, in general, is independent of structures formed in the mRNA.

51 On the other hand, recoding mechanisms are heavily reliant upon cis-acting RNA structures, such 52 as stem-loops, kissing loops, or pseudoknots, around stop codons ${ }^{14-16}$. However, the detailed 53 mechanisms by which such structured elements regulate recoding remain undefined. In the case 54 of readthrough, recoding signals are thought to stall ribosomes by acting as barriers to mRNA 55 unwinding, thus allowing for the recognition of near-cognate tRNAs during PRT ${ }^{17}$. The strained 56 unwinding also potentially acts as a mechanical stressor to force ribosomes into a new reading 57 frame during PRF ${ }^{15,17}$. 
The cis-acting, structured, recoding signals are mostly known to function autonomously.

60 Nevertheless, in some cases their folding can be manipulated by surrounding sequences to either

61 attenuate or enhance activity, and in rare cases they can be influenced by interaction with distal

62 downstream sequences. For example, potato leafroll virus (PLRV), carnation Italian ringspot

63 virus (CIRV), and barley yellow dwarf virus (BYDV) were all found to contain 3' elements,

64 located $700 \mathrm{nt}, 3.5 \mathrm{~kb}$, and $4 \mathrm{~kb}$, respectively, from the frameshifting signals that enhance recoding

65 via long-range base pairing interactions ${ }^{18-20}$. In even rarer cases, trans-acting protein factors have

66 been implicated in recoding. For example, the encephalomyocarditis virus (EMCV) has been

67 shown to require proteins A2 and porcine reproductive and respiratory syndrome virus (PRRSV)

68 to require $\mathrm{nsp} 1 \beta$ and $\operatorname{poly}(\mathrm{C})$ binding protein for proper recoding to occur ${ }^{21,22}$. It has been

69 suggested that these proteins, in complex with the RNA, substitute for the barriers usually

70 provided by structured signals ${ }^{18}$.

VEGF-A mRNA was shown to undergo a stop codon readthrough event to allow for a 22

73 amino acid extension (Fig. 1a) ${ }^{11}$. While VEGF-A164, the most common isoform, acts as a key

74 angiogenic agent ${ }^{23,24}$, the extended VEGF-Ax has been shown to be significantly less

75 angiogenic ${ }^{11,12}$. In the VEGF system, the 63 nucleotides which code for the Ax-extension

76 immediately downstream of the stop codon, were implicated as the signal required for ribosomal

77 stop-codon readthrough ${ }^{11}$. Furthermore, it was reported that the event also requires the

78 heterogeneous nuclear ribonucleoprotein (hnRNP) A2/B1 as a trans-acting factor ${ }^{11}$ in that

79 mutations to the hnRNP A2/B1 binding site on the Ax-element and siRNA knockdowns of the

80 protein itself lead to lower levels of stop-codon readthrough. It is important to note that a

81 subsequent study by Loughran, et al. failed to replicate the same read through capability of the

82 Ax-element ${ }^{25}$. Here we show that efficient readthrough in VEGF-Ax mRNA requires full-length

83 mRNA, with multiple cis-acting signals in the coding region working synergistically with each

84 other and with the trans-acting hnRNP A2/B1 to confer readthrough activity. 


\section{Results}

\section{VEGF-Ax readthrough is regulated by distal RNA elements located in the coding region.}

To characterize the VEGF-A system, we used a dual luciferase reporter assay in which various sequences of interest were cloned between renilla and firefly luciferases to determine stop codon readthrough efficiency ${ }^{26}$. First, the natural leakiness of the UGA stop codon was determined to be $\sim 0.05 \%$, which is in agreement with previous studies ${ }^{27}$ (Fig. 1b). Second, we tested the full bicistronic coding sequence of the VEGF-A mRNA (VEGF-A+Ax), where we observed $\sim 40$-fold increase in readthrough levels to $\sim 2.2 \%$, a level similar to many functionally active recoding systems ${ }^{1}$. To ensure that the observed increase over the natural leakiness of the UGA stop codon was robust and specific, we tested Annexin A2 mRNA (Fig. 1b), a gene with no known readthrough activity. This construct also only allowed for $\sim 0.05 \%$ basal leakiness of the stop codon, demonstrating that VEGF-A mRNA indeed has the propensity for stimulating readthrough events. Third, we tested just the 63 nucleotide Ax-element downstream of the stop codon that was previously identified as the signal being responsible for readthrough activity ${ }^{11}$. This region only led to a 4 -fold increase over natural leakiness with a $0.2 \%$ readthrough activity (Fig. 1b), which is similar to the results obtained by Loughran, et al. ${ }^{25}$. Taken together these data indicated that sequences upstream in the coding region influence the process of readthrough in VEGF-Ax, and that the Ax-element alone is only marginally active for readthrough.

In an effort to identify factors responsible for the above observations, we first probed for the presence of potential local enhancers by adding 50, 100 or 200 nucleotides upstream of the Ax-element (Extended Data Fig. 1a). This did not result in increasing the readthrough levels to that of the whole coding sequence, thus implying that there are no local enhancer sequences for the Ax-element, and that sequences very distal and upstream to the stop codon enhance readthrough. We then created truncated constructs in which nucleotides were removed from the 5' end of the mRNA. Surprisingly, deletion of the first 100 nucleotides dropped the readthrough activity by half ( 0.94\% readthrough), with a further drop to Ax-only levels at the 250-nucleotide deletion (Fig. 1b). Interestingly, sequence analysis of the upstream region within the first $100 \mathrm{nt}$ presented another potential hnRNP A2/B1 binding sequences (A93GGAGG). Altogether, this set 
114 of data demonstrate that the full, bicistronic VEGF-A mRNA is necessary for efficient 115 readthrough to occur and that the Ax-element is incapable of causing significant readthrough 116 without the presence of multiple signals in the coding region: one within the first 100 nucleotides 117 with a potential to bind hnRNP A2/B1 (SL-Au1), and the second between 200-250 nucleotides $118\left(\mathrm{Au}_{2}\right)$.

DMS-MaPseq reveals alternate structures in SL-Au1 and multi-domain organization of the Ax-

121 element.

To gain structural insights into the readthrough process, we chemically probed ${ }^{28}$ the 123 bicistronic native mRNA from the cells used for the luciferase assay by DMS-MaPseq. Overall, 124 the Ax-element folds into three, short stem loops (SL-Ax1-3) with five and four nucleotide linker 125 sequences in between them, respectively. This architecture is unusual in that most recoding 126 signals are made up of pseudoknots or single stem loops (Fig. 1c and Extended Data Fig. 1b). In 127 addition, the first seven nucleotides of the Ax-element form an additional stem loop with ten 128 nucleotides upstream of the stop codon. SL-Ax1 contains the previously identified hnRNP A2/B1 129 binding site with one of the consensus A ${ }_{588}$ GG motifs predicted to be positioned in the loop ${ }^{11}$.

DMS mapping of the 5' end of the mRNA showed that only residues 43-102 give rise to 132 ensembles that converge into a defined fold, which we term SL-Au1. SL-Au1 was predicted to 133 form a long stem loop capped with a CCA triloop and has four short helices (1a-1d) interspersed 134 with bulges (Fig. 1c and Extended Data Fig. 1c). In some structures, helix 1c either folded into a 135 stem with a register shift (helix $1 c^{\prime}$ ) or did not form at all and instead configured into a large 136 bulge, suggesting that helix 1c could possibly sample alternate arrangements (Extended Data Fig. 137 1c). The potential hnRNP A2/B1 binding sequence spans both helix $1 \mathrm{a}$ and $1 \mathrm{~b}$ at the $3^{\prime}$ end of SL138 Au1. Finally, characterization of $\mathrm{Au}_{2}$ (residues 200-250) also shows the potential to fold into 139 structured elements. However, the configurations of the structures in the ensembles varied 140 significantly, with sequences placed in a stem in one configuration ending up in a loop in the 141 other (Extended Data Fig. 1d); for this study, we thus only focused on the roles of SL-Au1 and the 142 Ax-element in stop codon readthrough. 
Ax and Au1 elements make long-range interactions.

We next synthesized the individual RNA domains by in-vitro transcription. Given that we observed an effect of the Au1 region on stop codon readthrough, we tested whether it is able

147 to interact directly with the Ax-element. To do so, we performed Isothermal Titration Calorimetry 148 (ITC) to check for potential long-range interactions between the elements. While titration of SL$149 \mathrm{Au}_{1}$ into SL-Ax 3 did not give rise to any binding interactions, titration of SL-Ax 2 into SL-Au1 gave rise to specific enthalpically-driven heats of binding between the two domains $\left(K_{d}=0.95 \pm 0.53\right.$ $\mu \mathrm{M})$ at a 1:1 stoichiometry $(\mathrm{n}=0.93 \pm 0.1)($ Fig. $1 \mathrm{~d})$. For comparison, a study by Vander Meulen $e t$ al. has characterized a GNRA tetraloop and receptor stem docking interaction - prevalent in maintaining long range structures in RNA molecules - to have an estimated dissociation constant of $\sim 5.5 \mu \mathrm{M}^{29}$. It is also important to note that in the Vander Meulen et al. study, the interaction was made bivalent by designing the tetraloop and receptor onto the same construct, which in all likelihood further enhanced the binding. Thus, the relatively strong affinity between SL-Au1 and SL-Ax2 suggests a model in which long-range interactions add an additional layer of complexity 158 to the VEGF-A readthrough event.

\section{Structures of the Ax-element.}

We then performed structural analysis by Nuclear Magnetic Resonance (NMR) to understand the individual motifs of the Ax-element (Extended Data Table 1). In SL-Ax1, the 5-bp 163 stem predicted by DMS-MaPseq is extended by an additional non-canonical A587 o G592 base pair, 164 thus resulting in a four-nucleotide A588GGA tetraloop (Fig. 1f and Extended Data Fig. 2a). The 165 first two residues (A $\mathrm{A}_{58}$ and G589) of the loop are in a stacked configuration with the 5' nucleotides of the helix, with G589 in a syn-conformation. After chain reversal, residue G590 also adopts a synconformation, stacking on A591, which further stacks continuously with the 3 ' nucleotides of the helix. Thus, for the two consensus AGG hnRNP A2/B1 binding sequences, A ${ }_{584} \mathrm{GG}$ is completely sequestered by base pairings in the stem, while the $\mathrm{A}_{588} \mathrm{GG}$ nucleotides are structured in an unusual tetraloop. 
As predicted, SL-Ax2 forms a four base pair stem capped with a $\mathrm{U}_{607} \mathrm{CGGG}$ pentaloop. The

173 first two residues of the pentaloop, $\mathrm{U}_{607}$ and $\mathrm{C}_{608}$, continue to stack on the $5^{\prime}$ strand of the stem

174 base pairs, after which the chain turns with the following three residues G ${ }_{609} \mathrm{GG}$ showing 175 continuously stacking NOES with the 3' strand of the helix (Fig 1g and Extended Data Fig. 2b).

176 As in the GNRA-type fold, the nucleobase of G611 stacks on the ribose of A612 leading to an 177 expected upfield shift of the $\mathrm{A}_{612} \mathrm{H}^{\prime}$ proton to $4.5 \mathrm{ppm}$ (Extended Data Fig. 2c). This positions 178 the Watson-Crick faces of the three contiguous G residues outside the loop. Such a loop 179 configuration where three stacked $G$ residues engage in Watson-Crick interactions with 180 contiguous cytosines present at a long-distance has been observed in the Haloarcula marismortui 181 ribosomal $\mathrm{RNA}^{30}$. Thus, our structure of SL-Ax2 indicate that it may be poised for the long-range 182 interactions with structures present in $\mathrm{Au} 1$ as evidenced by ITC analysis.

Finally, the imino proton assignments of SL-Axз indicate that it is composed of a five base pair stem-loop with a dinucleotide $\mathrm{A}_{632} \mathrm{~A}$ bulge, as predicted by DMS-MaPseq. Continuous stacking of the five base pairs was observed, indicating that the AA bulge is extruded out of the groove (Extended data Fig. 2d). However, dimerization interactions of the loop precluded us from unambiguously assigning and solving the high-resolution structure of SL-Ax3.

\section{Structure of the Au1 element.}

To aid in assignments of the relatively large $\mathrm{Au}_{1}$ construct and to unambiguously corroborate the presence of the equilibrium structure predicted in the SL-Au1 element, we synthesized smaller segments of $\mathrm{Au}_{1}\left(1 \mathrm{a}-1 \mathrm{~b}, 1 \mathrm{~b}-1 \mathrm{c}, 1 \mathrm{~b}-1 c^{\prime}, 1 c^{\prime}-1 \mathrm{~d}, 1 \mathrm{c}-1 \mathrm{~d}\right.$ and $1 \mathrm{~d}$; along with the 194 full-length construct (Extended Data Fig. 3-6). Indeed, our data show that SL-Au1 forms a single 195 stem with a CCA loop and is interspersed with bulges that divide the stem into four short helices, 1a-1d (Extended Data Fig. 3). Furthermore, as predicted, two sets of signals were assigned for the

197 region encompassing residues 56-64 and 81-90, which confirmed an equilibrium between helix 1c 198 and 1c' (Fig. 1c and Extended Data Fig. 6a). 
The structures formed by helices $1 \mathrm{a}, 1 \mathrm{~b}$ and $1 \mathrm{~d}$ do not change in the two configurations adopted by SL-Au1 (Extended Data Table 1). Separated by a single adenine nucleotide bulge (A50), 202 the distal helices 1a and 1b form an almost continuous stem (Fig. 1e and Extended Data Fig. 4a, $2034 \mathrm{~b}) . \mathrm{A}_{50}$ is part of the helix with regular stacking between residues $\mathrm{U}_{49}$ and $\mathrm{C}_{51}$. In this 204 configuration, the two consensus hnRNP A2/B1 binding sequences, A93GG and A96GG, are all 205 involved in base pairing interactions within helix $1 \mathrm{~b}$ and 1a, respectively (Extended Data Fig. 3). 206 Furthermore, the proximal helix 1d is rich in GU basepairs ( $\mathrm{U}_{67}-\mathrm{G}_{79}, \mathrm{G}_{68}-\mathrm{U}_{78}$ and $\left.\mathrm{U}_{70}-\mathrm{G}_{76}\right)$, which 207 make up half of the six base pairs in the helix as evidenced by the three upfield shifted GU cross208 peak pairs in the imino region of the NOESY spectrum (Fig. 1e and Extended Data Fig. 5b). The $209 \mathrm{C}_{72} \mathrm{CA}$ triloop which caps this helix is well structured, with chain reversal occurring between residues $\mathrm{C}_{72}$ and $\mathrm{C}_{73}$ and stacking with both sides of the helix (Fig. 2a).

214 tandem, non-canonical C-C base pairs that are protonation dependent, making the equilibrium 215 between helix 1c and $1 c^{\prime}$ highly sensitive to $\mathrm{pH}$ (Fig. $2 \mathrm{~b}$ and Extended Data Fig. 5b). In the 1c 216 configuration, the junction between helices 1d and 1c also has an almost continuously stacking 217 architecture with a single adenine nucleotide bulge ( $\left.\mathrm{A}_{65}\right)$ stacked inside the helix. On the other 218 end, the junction between $1 \mathrm{c}$ and $1 \mathrm{~b}$ is defined by an internal loop $\left(\mathrm{A}_{56} ; \mathrm{C}_{89} \mathrm{~A}\right)$. While $\mathrm{A}_{56}$ stacks 219 with the helix (Extended Data Fig. 4b), the opposing residue A90 is extruded out toward the minor 220 groove. The $\mathrm{H}_{4}$ ' sugar proton of residue $\mathrm{C}_{89}$ gives NOEs to the aromatic $\mathrm{H} 8$ proton of A92, placing

221 it in the major groove and within hydrogen bonding distance of $\mathrm{U}_{54}-\mathrm{A}_{92}$ (Fig. 2c). This local triple222 base configuration obtained is similar to arginine sandwich motifs ${ }^{31}$. Overall, with continuous 223 stacking of all helices in the helix 1c configuration, the structure of SL-Au1 has a linear appearance 224 (Fig. 1e).

On the other hand, due to the base pairing register shift in the helix $1 c^{\prime}$ configuration, 227 residues $A_{56}$ to $A_{59}$ and $A_{81}$ to $A_{85}$ form two opposing bulges separated by a 5-bp stem (residues 228 60-65 and 86-90). These four (A ${ }_{56} \mathrm{CCA}$ ) and five (A $\left.{ }_{81} \mathrm{CCCA}\right)$ nucleotide bulges flanking helix 1c' 
cause bent geometries between junctions with helices $1 \mathrm{~b}$ and $1 \mathrm{~d}$, respectively (Fig. $2 \mathrm{~d}$ ). At the $1 \mathrm{~b}$ $1 c^{\prime}$ junction, residues $A_{56}$ and $C_{57}$ stack on helix $1 b$, whereas residues $C_{58}$ and $A_{59}$ stack under helix

$2311 c^{\prime}$ (Fig. 2e). There are no NOEs between $C_{57}$ and $C_{58}$, indicating opposing orientations. At the $1 c^{\prime}-$

$2321 \mathrm{~d}$ junction, the $\mathrm{A}_{81}$ in the $\mathrm{A}_{81} \mathrm{CCCA}$ bulge base pairs with the $\mathrm{A}_{65}$ in the opposite strand (Fig. 2f).

233 The following three cytosine residues show a continuous NOE walk. With only a partial stacking

234 between $\mathrm{C}_{84}$ and $\mathrm{A}_{84}$, the triplet $\mathrm{Cs}$ are extruded out in way that seem poised to make long-range

235 interaction with SL-Ax2, as indicated by the ITC data (Fig. 1d, 2d).

\section{SL-Ax 2 and SL-Au interact via loop-bulge docking interactions}

To understand the mode of the long-range interaction, we performed small angle X-ray scattering (SAXS) analysis of free SL-Au1 and in complex with SL-Ax2. Furthermore, since the

$240 \mathrm{C}_{82} \mathrm{CC}$ motif in SL-Au1 and $\mathrm{G}_{609} \mathrm{GG}$ motif in SL-Ax2 resemble a motif found in Haloarcula

241 marismortui ribosomal RNA where the three stacked G residues engage in Watson-Crick 242 interactions with contiguous cytosines ${ }^{30}$, we also modeled a similar interaction between SL-Au1 243 and SL-Ax2 (Fig. 2g). Compared to the free form, the SAXS envelop of the bound form of SL-Au 1 244 showed a clear additional density, which was able to accommodate the structural model of SL$245 \quad$ Ax2 in the complex (Fig. 2g).

246 To unambiguously assign the docking interface, we titrated SL-Ax 2 in the $1 \mathrm{c}^{\prime}-1 \mathrm{~d}$ construct, 247 which allowed for identification of the $\mathrm{A}_{81}-\mathrm{C}_{84}$ bulge as the one involved in the long-range 248 interaction (Extended Data Fig. 5b). While no global changes in the secondary structure of either $2491 c^{\prime}-1 d$ or SL-Ax2 occurred, resonances for three new G-C Watson-Crick base pair formations were 250 observed, supporting complex formation observed by ITC (Fig. 1d). The combination of binding 251 heats observed by ITC, intermolecular base pairing NOEs observed by NMR, and the complex 252 obtained by SAXS all support the formation of the long-range interaction between the SL-Au1 253 bulge and SL-Ax2 loop.

\section{hnRNP A2/B1 binds SL-Ax 1 and SL-Au1}

We next wanted to test if the previously identified hnRNP A2/B1 binding sites (A ${ }_{584} \mathrm{GG}$ 257 and $\mathrm{A}_{588} \mathrm{GG}$ in $\mathrm{SL}-\mathrm{Ax}_{1}$ ) and the new potential sites ( $\mathrm{A}_{93} \mathrm{GG}$ and $\mathrm{A}_{96} \mathrm{GG}$ in helix $1 \mathrm{a}$ and 1b, 
respectively) ${ }^{11}$ interacted with hnRNP A2/B1. It was previously reported that mutating the A ${ }_{58}$ GG hnRNP A2/B1 binding site led to decreased readthrough levels ${ }^{11}$. Similar to previous

260 hnRNP binding studies ${ }^{32-36}$, we used an hnRNP construct that consisted of the two RNA-

261 recognition motifs (RRMs), but lacked the aggregation-prone C-terminal domain, previously 262 shown to be involved in oligomerization and nuclear localization of the protein ${ }^{37-39}$ (Fig. 3a).

263 Titration of hnRNP A2/B1 into SL-Ax 1 and SL-Au1 by ITC gave data that fit well with a single-site 264 binding model with estimated dissociation constants of $200 \pm 42 \mathrm{nM}$ and $348.5 \pm 22 \mathrm{nM}$, 265 respectively ( $\mathrm{n}=1.02 \pm 0.34$ and $1.2 \pm 0.33$, respectively) (Fig. $3 b)$. These $\mathrm{K}_{\mathrm{d}}$ 's are in accordance with 266 previously published binding affinity of similar sequences ${ }^{36}$. Nevertheless, a critical difference in 267 the binding mode was observed between the two interactions: while the interaction with SL-Ax1 268 was enthalpically driven $(\Delta \mathrm{H}-10.4 \pm 4.7 \mathrm{kcal} / \mathrm{mol}$ and $\Delta S-4.4 \pm 15.3 \mathrm{cal} / \mathrm{mol} / \mathrm{K})$, the interaction 269 with SL-Au $1(\Delta \mathrm{H}-3.6 \pm 1.7 \mathrm{kcal} / \mathrm{mol}$ and $\Delta S 17.6 \pm 5.7 \mathrm{cal} / \mathrm{mol} / \mathrm{K})$ was entropically driven, the latter 270 suggesting rearrangement of RNA structure upon protein binding.

Since hnRNP A2/B1 bound both SL-Au1 and SL-Ax1, and since it is able to dimerize 273 through its C-terminal domain ${ }^{38}$, we tested if it is able to mediate the long-range interaction 274 between SL-Au1 and SL-Ax1 via its dimerization domain. Using an in-vitro rabbit reticulocyte 275 lysate (RLL) system, we tested the effects of full-length hnRNP A2/B1 and hnRNP A2/B1 lacking 276 a C-terminal domain $(\triangle \mathrm{CTD})$ on VEGF-A readthrough efficiencies (Fig. 3c). Recoding was 277 replicated in this assay, with baseline readthrough values of $\sim 4 \%$, which is slightly higher than 278 those in cells. Such differences have previously been reported ${ }^{40}$, and generally arise from tighter 279 regulation in cells. By adding either full-length hnRNP A2/B1 or hnRNP A2/B1- $\Delta$ CTD to VEGF280 A mRNA, we are able to increase readthrough by a factor of $\sim 2.5$ (Fig. 3c) to 9.6\%, and further 281 additions of hnRNP A2/B1 reproducibly reduced readthrough. These results not only confirm the 282 stimulatory effect of hnRNP A2/B1 binding, but also point to other hnRNP A2/B1 driven 283 mechanisms to balance required readthrough levels. Importantly, there was no observable 284 difference in readthrough levels between the full-length and dimerization-deficient hnRNP 285 A2/B1 constructs, indicating that hnRNP A2/B1 dimerization is not required for readthrough 286 activity, and that another mechanism must be responsible for mediating the observed long-range 
287

288

289

290

291

292

293

294

295

296

297

298

299

300

301

302

303

304

305

306

307

308

309

310

311

312

313

314

interactions. As a control, using the Murine Leukemia Virus (MLV) readthrough system resulted in constant readthrough levels, showing that the effects of hnRNP A2/B1 are specific to VEGF-A (Fig 3c).

To determine the exact binding site within SL-Au1, we performed a protein titration on our NMR sample. Addition of even sub-stoichiometric (0.3 equivalents) of RRM1/2 to SL-Au1 resulted in pushing the equilibrium to the $1 c^{\prime}$ configuration, as evidenced by the disappearance of the $\mathrm{U}_{60}-\mathrm{A}_{85}$ and $\mathrm{A}_{59}-\mathrm{U}_{86}$ base pairing in helix 1c and the appearance of $\mathrm{A}_{64}-\mathrm{U}_{86}$ in helix 1c' (Fig. 3d). Furthermore, addition of one equivalent of protein led to chemical shift changes both in the protein and RNA and gave rise to multiple intermolecular NOEs. Interestingly, our data show that binding occurred specifically to molecules in the $1 c^{\prime}$ configuration, as evidenced by the specific disappearance of the $\mathrm{U}_{60}-\mathrm{A}_{90}$ base pair, which is representative of helix $1 c^{\prime}$. Perturbations of $\mathrm{A}_{93}$ resonances indicated that binding occurs at the consensus hnRNP A2/B1 binding site (Fig. 3d). Overall, these experiments suggest that the role of hnRNP A2/B1 binding to SL-Au1 may be to modulate the equilibrium toward the $1 c^{\prime}$ conformation, which is required for long-range interactions.

Based on recent findings that it is the RRM1 that recognizes the AGG sequence ${ }^{36}$, we used a shortened hnRNP construct to determine the mode of interaction with SL-Ax1. Binding between RRM1 and SL-Ax1 resulted in perturbations of specific residues in the loop, with G590 giving clear intermolecular NOEs to an aromatic residue of RRM1, thus providing confirmation of residues A ${ }_{588}$ GG sequence as the binding site (Fig. 3e). The second potential A ${ }_{584} \mathrm{GG}$ binding motif remains sequestered within the stem as unaffected by protein binding; in fact, the majority of the SL-Ax 1 stem structure remaining unchanged upon protein addition. Thus, at both the SL-Au1 and SL-Ax 1 binding sites, hnRNP A2/B1 recognizes structured loops, as recently described in viral IRES translation regulation ${ }^{35}$.

\section{The various elements synergistically regulate recoding efficiencies}


Given both the unusual multi-stem nature of the Ax-element, and potential unique functions of the individual stems, we wanted to first determine their contributions to recoding.

317 We created constructs in which SL-Ax 2 and SL-Ax 3 elements were removed (Fig. 4a). We also 318 checked DMS-MaPseq reactivities to ensure that in either deletion, the fold of SL-Ax $x_{1}$ is not 319 perturbed (Extended Data Fig. 7a). Deletions of SL-Ax $x_{3}$ and both SL-Ax $x_{2}$ and SL-Ax 3 led to 320 significant reduction in readthrough levels by $40 \%$ and $70 \%$, respectively, implying that the 321 entirety of the Ax-element is required to maximize readthrough levels (Fig. 4b). Interestingly, 322 removing the entire Ax-element decreased readthrough levels by almost $80 \%$ to a level of $0.5 \%$ 323 (Fig. 4b).

While we do not yet understand the structural mechanism by which SL-Ax 3 influences readthrough, our understanding of the long-range interaction between SL-Ax 2 with SL-Au1 327 allows us to test for its contribution. We thus created mutants in which the $\mathrm{C}_{82} \mathrm{CC}$ involved in 328 long range interaction in SL-Au1 was sequestered by either changing them to Gs along with 329 mutating $\mathrm{G}_{61}$ to $\mathrm{C}$, or by mutating the opposing $\mathrm{C}_{62} \mathrm{C}$ residues to guanosines. Both of these give 330 rise to three contiguous G-C base pairs, which should preclude inter-domain interaction of this 331 bulge and lead to appreciable decreases in readthrough by $\sim 40 \%$ and $\sim 50 \%$, respectively (Fig. $4 \mathrm{c}$ ). 332 Similarly, disruption of the docking G $609 \mathrm{GG}$ motif in SL-Ax2 by replacing the pentaloop with a 333 GAAA tetraloop, led to an equivalent $40 \%$ decrease in readthrough activity (Fig. 4c).

Next, we wanted to test the contributions of protein binding motifs to recoding efficiencies. We first tested protein binding efficiency of the previously published dinucleotide 337 A 587 A to $\mathrm{U}_{587} \mathrm{U}$ mutation in SL-Ax ${ }_{11}{ }^{11}$. ITC experiments on a SL-Ax 1 A587 A:UU mutant yielded a $338 \sim 1.5$-fold decrease in binding when compared to wild-type SL-Ax1 (Extended Data Fig. 7b). This correlates with an observed $\sim 50 \%$ reduction in readthrough levels (Fig. 4c). Similarly, for experiments on a SL-Au1 $\mathrm{A}_{92} \mathrm{~A}: \mathrm{UU}$ mutant, we observed a $\sim 2$-fold increase in $\mathrm{K}_{\mathrm{d}}$, or 2-fold decrease in binding affinity (Extended Data Fig. 7c), which correlates to a $\sim 40 \%$ decrease in

342 readthrough levels for this mutation (Fig. 4c), suggesting a strong-interplay between hnRNP 343 A2/B1 binding and readthrough of the VEGF-Ax system. Interestingly, a double mutant in which 
344 both hnRNP A2/B1 binding sites were mutated displayed higher readthrough efficiency than 345 either single mutant (Fig. 4c). This epistatic behavior is a strong indication of direct influence of 346 the two binding events on local RNA structure, and consequently their long-range interactions. 347 Furthermore, it suggests that the closed-loop configuration of VEGF mRNA may place SL-Au1 348 and SL-Ax 2 in close proximity, thus allowing for the observed epistasis. Overall, these mutational 349 studies show the importance of the various players involved in regulating readthrough levels of 350 the VEGF mRNA. 


\section{Discussion}

352 Here we have presented evidence of a complex eukaryotic translational readthrough 353 system employing several novel mechanisms of action. Canonically, readthrough and 354 frameshifting events only require a single cis-acting signal in the vicinity of the stop codon. In this 355 study, we performed structural and mutational work to better understand the role of the 356 previously identified readthrough signal, the Ax-element. In addition to the previously identified 357 stop-codon proximal signal, we identified several new components of this readthrough event: 358 two cis-acting elements upstream of the stop codon, an additional hnRNP A2/B1 binding site, and long-range RNA-RNA interactions spanning the length of the VEGF-A gene.

The presence of a $5^{\prime}$ element conferring ribosomal readthrough is novel. In retroviruses,

362 where ribosomal recoding has been studied more extensively, readthrough signals tend to be 363 localized in the immediately vicinity of the stop codon ${ }^{14,16}$. While distant downstream elements 364 enhancing frameshifting in Pea enation mosaic virus ${ }^{41}$ and readthrough in Potato leafroll virus ${ }^{20}$ 365 have been studied, to the best of our knowledge, there are no examples of distant upstream 366 signals. Given that these novel cis-acting elements, located at $\sim 250$ and $~ 500$ nucleotides upstream 367 of the stop codon, are in the coding region, this is an even more unusual setup for ribosomal 368 readthrough. The dispersed, multi-domain architecture of the Ax-element is also unique in that 369 RNA signals that cause recoding in ribosomes are generally made up of a single stem loop or 370 pseudoknot structures. Such an organization, along with the presence of long-range interactions 371 between signals located at the two ends of the coding sequence and multiple hnRNP A2/B1 372 binding sites, hints towards a complex mechanism for regulation of readthrough in mammalian 373 systems.

Altogether, our study allows us to start putting together a mechanism by which

376 readthrough frequency - and hence relative proportions of VEGF-A (angiogenic) to VEGF-Ax 377 (less angiogenic) isoforms - may be maintained (Fig. 5). Our model suggests that regulation 378 takes place via modulations of alternate mRNA structures by hnRNP A2/B1 levels ${ }^{42}$. Binding of 379 hnRNP A2/B1 to SL-Au1 allows for a register shift in the base pairing of helix 1c to the $1 c^{\prime}$ 
configuration, which is capable of making long-range interactions with SL-Ax2 near the vicinity

381 of the stop codon. The two conformations of SL-Au1 were reproducibly observed in cells, adding 382 another example of use of conformational equilibrium in RNA molecules to regulate critical 383 functions in cells. Additionally, while we did not address the roles of SL-Axз or the Au2 element $384 \sim 250 \mathrm{nt}$ upstream of the stop codon in this manuscript, they will further contribute to the 385 complexity of this readthrough system.

Whereas the function of hnRNP A2/B1 binding to SL-Au1 is to modulate the equilibrium, the function of hnRNP A2/B1 binding to SL-Ax 1 is unclear. However, given the spacing between the stop codon and the hnRNP A2/B1 binding site at SL-Ax1, the binding of hnRNP A2/B1 may act as a stressor upon the ribosome at the stop codon. This would be similar to EMCV and PRRSV

391 in which binding of $\mathrm{A} 2$ protein and $\mathrm{nsp} 1 \beta / \operatorname{poly}(\mathrm{C})$ binding protein, respectively, takes the place 392 of the canonical structured element ${ }^{21,22}$. Given the apparent lack of an extended, complex secondary structure in VEGF-A mRNA, this hnRNP A2/B1-induced stress, in conjunction with

394 the additive effects of the long-range RNA interaction, may take the place of complex secondary structural elements, such as pseudoknots, found in many other recoding events ${ }^{15}$.

The long-range cross-talk interaction we have identified is a novel mechanism through which ribosomal recoding events may be regulated. While the broader concept of long-range RNA interactions is not new, it has recently been suggested that more broadly, most if not all mRNAs exhibit $5^{\prime}$ to $3^{\prime}$ communication through a combination of proximity base pairing and RNA-binding proteins ${ }^{43,44}$. For example, recent studies measuring the spacing between $5^{\prime}$ and $3^{\prime}$ ends of coding mRNA, both in-vitro and in-vivo, have found them to be much closer than would

403 be predicted for a randomly folded RNA ${ }^{43,45-47}$. While these studies support a previously 404 established closed-loop model of RNA in which the polyA tail and 5' cap of mRNA are spatially 405 close to regulate canonical translation initiation and termination, our studies show that the 406 closed-loop model can give rise to specific long-range interactions that can have functional 407 consequences on translational elongation. 
Sequences of interest for in-cellulo dual luciferase assays were cloned between renilla

411 luciferase (RLuc) and firefly luciferase (FLuc) using BamHI and SacI restriction sites on the p2Luc

412 plasmid $^{26}$. To normalize against differential FLuc and RLuc luminescence, mRNA degradation,

413 and ribosome fall-off products, each construct containing a TGA stop codon was cloned in 414 tandem with a control construct containing a GCA codon. This control construct was used to 415 normalize for readthrough activity.

Dual luciferase assays were performed in white 96-well half-area flat bottom plates 418 (Corning). Bovine aortic endothelial cells (BAOECs), grown at $37^{\circ} \mathrm{C}$ in $5 \% \mathrm{CO}_{2}$, were diluted to $41930 \%$ confluency in DMEM supplemented with 10\% FBS and 1\% penicillin-streptomycin. Cells 420 were then plated at $150 \mu \mathrm{L}$ per well with 5 total replicates per construct. 1 hour post-plating, 11 $\mu \mathrm{L}$ 421 transfection mix (480 ng plasmid, $1.2 \mu$ L Fugene6 (Promega) and 46.8 $\mu \mathrm{L}$ DMEM) was added to 422 each well. Cells were allowed to express RLuc and FLuc for 2 days prior to taking readings.

Luciferase luminescence readings were collected on a SpectramaxL (Molecular Devices) 425 plate reader at $470 \mathrm{~nm}$ with an integration time of 1 second using the reagents in the Dual426 Luciferase Reporter Assay System kit (Promega). Prior to collection, cells were lysed in $35 \mu \mathrm{L}$ of 427 1x passive lysis buffer (Promega) for 15 minutes. Then $50 \mu \mathrm{L}$ FLuc substrate was added and 428 allowed to sit for 30 seconds prior to collecting luminescence readings. Similarly, 50 $\mu$ L RLuc 429 substrate was added, allowed to sit, and luminescence was collected again. Readthrough 430 percentages were calculated as previously described ${ }^{26}$. P-values were calculated using a 2-tailed 431 T-test.

\section{In-vitro translation assay}

434 Template DNA for rabbit reticulocyte lysate (RRL) assays was prepared by PCR 435 amplifying hnRNP A2/B1 constructs and VEGF-A sequences of interest flanked by RLuc and 436 FLuc. $8 \mu \mathrm{L}$ PCR product was used as a template for in-vitro transcription using the mMESSAGE 
437 mMACHINE T7 Transcription Kit (Invitrogen). RNA was diluted to $10 \mu \mathrm{g} / \mu \mathrm{L}$ in nuclease free 438 water.

$4412.5 \mu \mathrm{L}$ methionine) was combined with $1 \mu \mathrm{L}$ of each RNA (VEGF and hnRNP A2/B1) at the 442 desired concentration. For no protein samples, $1 \mu \mathrm{L}$ transcription reaction buffer was added 443 instead. Reactions were then allowed to continue for 90 minutes at $30^{\circ} \mathrm{C}$ prior to placing on ice 444 for 20 minutes. Lysates were then plated at $2.5 \mu \mathrm{L}$ per well with 3 total replicates per construct in 445 white 96-well half-area flat bottom plates (Corning). Luciferase luminescence readings were 446 collected as described above.

\section{Preparation of hnRNP A2/B1}

An hnRNP A2/B1 consisting of RRM1/2 was cloned into a pGEX-6P-1 plasmid using

451 BamHI and NotI restriction sites ${ }^{48}$.

BL-21 cells containing GST-tagged hnRNP A2/B1 constructs in pGEX-6P-1 were 454 inoculated in LB media containing $100 \mu \mathrm{g} / \mathrm{mL}$ ampicillin and grown overnight at $37^{\circ} \mathrm{C}$ with 455 shaking at $200 \mathrm{rpm}$. In the morning, $20 \mathrm{~mL}$ of overnight culture were diluted into $2 \mathrm{~L}$ LB media 456 containing $100 \mu \mathrm{g} / \mathrm{mL}$ ampicillin. Cells were allowed to grow at $37^{\circ} \mathrm{C}$ with shaking until reaching 457 OD $600 \sim 1$. Cells were cooled to $20^{\circ} \mathrm{C}$ and subsequently induced with $100 \mu \mathrm{M}$ IPTG for 16 hours, 458 after which they were pelleted at 5,000g for 20 minutes. A pellet corresponding to $2 \mathrm{~L}$ was 459 resuspended in $50 \mathrm{~mL}$ hnRNP lysis buffer (50 mM Phosphate pH 7.5, $200 \mathrm{mM} \mathrm{NaCl}, 40 \mathrm{mM}$ $460 \mathrm{MgCl}_{2}, 1 \mathrm{mM}$ DTT, 0.1\% Triton X-100) and sonicated (Branson Sonifier 450) at setting 7-8 with $46150 \%$ amplitude for 6 pulses of 60 seconds each. $5 \mathrm{~mL}$ of $4 \%$ polyethyleneimine (PEI) was added 462 drop wise to the lysed cells and allowed to mix on ice for 30 minutes. The lysed cells were then 463 spun at $27,000 \mathrm{~g}$ for 20 minutes. 
Lysate supernatant was added to Pierce Glutathione Agarose Affinity Purification Media (Thermo Scientific) and allowed to bind for 2 hours on a rocker at $4^{\circ} \mathrm{C}$. Following binding, beads 467 were washed twice with $50 \mathrm{~mL}$ ice cold hnRNP wash buffer (50 mM Phosphate pH 7.5, $500 \mathrm{mM}$ $468 \mathrm{NaCl}, 1 \mathrm{mM}$ DTT). Subsequently, protein-bound beads were loaded onto a column and washed 469 overnight with $2 \mathrm{~L}$ hnRNP wash buffer using a pump at a flow rate of $1.8 \mathrm{~mL} / \mathrm{minute}$ at $4^{\circ} \mathrm{C}$. 470 Protein was eluded in $50 \mathrm{~mL}$ elution buffer (20 mM Phosphate pH 7.5, $15 \mathrm{mM} \mathrm{KCl,} 2 \mathrm{mM} \mathrm{MgCl}$, $4715 \%$ glycerol) and $2.4 \mathrm{mg}$ PreScission protease. The column was allowed to rock at $4^{\circ} \mathrm{C}$ for 8 hours 472 prior to collecting the first elution. hnRNP A2/B1 was concentrated in Ultra Centrifugal Filter 473 Units (Amicon) with a $3 \mathrm{kDa}$ cutoff and washed into the appropriate buffer as needed.

475 Large-scale synthesis and purification of RNA

RNA constructs were either ordered (IDT or Horizon Discovery) or synthesized in-house 477 using T7 polymerase as previously described ${ }^{49}$. Final RNA was washed into appropriate buffer as needed using Ultra Centrifugal Filter Units (Amicon).

NMR \& Structure Calculations

RNA samples were placed in $300 \mu \mathrm{L}$ NMR buffer $(10 \mathrm{mM}$ Tris $\mathrm{pH} 5.6,10 \mathrm{mM} \mathrm{NaCl}$ or 10 mM HEPES pH 5.6, $50 \mathrm{mM} \mathrm{KCl}$ ) in $\mathrm{D}_{2} \mathrm{O}$. For assignments of exchangeable proton and nitrogen resonance NOESY and HSQC were collected on samples lyophilized and resuspended in $\mathrm{H}_{2} \mathrm{O}$ supplemented with $10-15 \% \mathrm{D}_{2} \mathrm{O}$ at $280 \mathrm{~K}$. For non-exchangeable proton and carbon assignments spectra were collected in $99.96 \%$ D2O. Assignments were done with the use of NMRFx ${ }^{50}$. As previously described, CYANA ${ }^{51}$ was used to calculate initial structures of the SL-Au1, SL-Ax1 and SL-Ax2 with manually assigned restraints. Standard torsion-angle $\left( \pm 50^{\circ}\right.$ deviations from ideality) and cross-helix phosphate-phosphate restraints were used for $\alpha$-helical regions. The structure with the lowest target function was used to create a coordinate file for refinement in xplor-nih ${ }^{52,53}$.

490 Phosphate- phosphate distances were not used at this step. Molecular images were generated 491 with PyMOL (http:// www.pymol.org). 
Most ITC experiments were performed in ITC buffer (10 mM HEPES pH 8.0, $50 \mathrm{mM} \mathrm{KCl,}$

$4951 \mathrm{mM}$ EDTA, $1 \mathrm{mM}$ BME), with the exception of certain SL-Ax1 constructs (20 mM phosphate $\mathrm{pH}$ $4967.2,15 \mathrm{mM} \mathrm{KCl}, 2 \mathrm{mM} \mathrm{MgCl}$, 5\% glycerol). RNA constructs were diluted to $5 \mu \mathrm{M}$ for and hnRNP 497 A2/B1 constructs were diluted to $100 \mu \mathrm{M}$. ITC experiments were performed on an ITC-200 498 microcalorimeter (MicroCal) by loading RNA into the cell and protein into the syringe. Protein 499 was injected in $2 \mu \mathrm{L}$ increments for a total of 20 injections. Baseline correction, binding curve 500 fitting, and parameter calculation were performed using Origin (OriginLab).

501

502 DMS-MaPseq

503 In-cellulo DMS-MaPseq samples were prepared by seeding 10-cm cell culture dishes of 504 BAOECs at 30\% confluency. 1 hour post-plating, 660 $\mathrm{L}$ transfection mix (5 $\mu$ g plasmid, $26 \mu \mathrm{L}$ 505 Fugene6 (Promega) and $650 \mu \mathrm{L}$ DMEM) was added to each well and allowed to grow for 2 days. 506 Sample processing and analysis was performed as previously described ${ }^{28,54}$.

508 Small Angle X-Ray Scattering

509 SAXS experiments were performed at the SIBYLS beamline (Advanced Light Source, 510 Lawrence Berkeley National Laboratory $)^{55}$. Samples were prepared in NMR buffer (10 mM Tris $511 \mathrm{pH}$ 5.6, $10 \mathrm{mM} \mathrm{NaCl}$ ) supplemented with 2\% glycerol. Data analysis and ab initio envelope 512 reconstruction were done using ScÅtter software ${ }^{56}$. 


\section{Figures/Tables}

Extended Data Table 1- NMR statistics and restraints

NMR and refinement statistics for the $\mathrm{Au}_{1}$ region

\begin{tabular}{|c|c|}
\hline & $\mathbf{A} \mathbf{u}_{1}$ \\
\hline \multicolumn{2}{|l|}{ NMR distance and dihedral constraints } \\
\hline \multicolumn{2}{|l|}{ Distance restraints } \\
\hline Total NOE & 463 \\
\hline Intraresidue & 297 \\
\hline Inter-residue & 166 \\
\hline Sequential $(|i-j|=1)$ & 164 \\
\hline Nonsequential $(|i-j|>1)$ & 2 \\
\hline Hydrogen bonds & 127 \\
\hline Total dihedral-angle restraints & 1014 \\
\hline Base pair & 0 \\
\hline Sugar pucker & 240 \\
\hline Backbone & 774 \\
\hline Based on A-form geometry & 1014 \\
\hline \multicolumn{2}{|l|}{ Structure statistics } \\
\hline \multicolumn{2}{|l|}{ Violations (mean \pm s.d.) } \\
\hline Distance constraints $(\boldsymbol{\Lambda})$ & $2.8 \pm 0.4$ \\
\hline Dihedral-angle constraints $\left(^{\circ}\right)$ & $41.0 \pm 3.5$ \\
\hline \multicolumn{2}{|l|}{ Deviations from idealized geometry } \\
\hline Bond lengths $(\boldsymbol{\Lambda})$ & $0.005 \pm 0.000$ \\
\hline Bond angles $\left({ }^{\circ}\right)$ & $0.628 \pm 0.006$ \\
\hline Impropers $\left({ }^{\circ}\right)$ & $0.352 \pm 0.009$ \\
\hline \multicolumn{2}{|c|}{ Average pairwise r.m.s. deviation $(\AA)^{a}$} \\
\hline All RNA heavy & 0.357 \\
\hline All nucleotides & 0.3717 \\
\hline
\end{tabular}

$\mathrm{NMR}$ and refinement statistics for the $\mathrm{Ax}_{1}$ region

\begin{tabular}{|c|c|}
\hline & $\mathbf{A x} \mathbf{x}_{1}$ \\
\hline \multicolumn{2}{|c|}{ NMR distance and dihedral constraints } \\
\hline \multicolumn{2}{|l|}{ Distance restraints } \\
\hline Total NOE & 123 \\
\hline Intraresidue & 74 \\
\hline Inter-residue & 49 \\
\hline Sequential $(|i-j|=1)$ & 45 \\
\hline Nonsequential $(|i-j|>1)$ & 4 \\
\hline Hydrogen bonds & 37 \\
\hline Total dihedral-angle restraints & 272 \\
\hline Base pair & $\mathbf{0}$ \\
\hline Sugar pucker & 64 \\
\hline Backbone & 208 \\
\hline Based on $\mathrm{A}$-form geometry & 272 \\
\hline \multicolumn{2}{|l|}{ Structure statistics } \\
\hline \multicolumn{2}{|l|}{ Violations (mean \pm s.d.) } \\
\hline Distance constraints ( $\boldsymbol{A})$ & $0.6 \pm 0.5$ \\
\hline Dihedral-angle constraints $\left({ }^{\circ}\right)$ & $11.8 \pm 2.1$ \\
\hline \multicolumn{2}{|l|}{ Deviations from idealized geometry } \\
\hline Bond lengths $(\AA)$ & $0.004 \pm 0.000$ \\
\hline Bond angles $\left({ }^{\circ}\right)$ & $0.667 \pm 0.007$ \\
\hline Impropers $\left({ }^{\circ}\right)$ & $0.369 \pm 0.009$ \\
\hline \multicolumn{2}{|c|}{ Average pairwise r.m.s. deviation $(\hat{\Lambda})^{a}$} \\
\hline All RNA heavy & 0.0687 \\
\hline All nucleotides & 0.4137 \\
\hline
\end{tabular}

NMR and refinement statistics for the $\mathrm{Ax}_{2}$ region

\begin{tabular}{ll}
\hline & $\mathrm{Ax}_{2}$ \\
\hline NMR distance and dihedral constraints & \\
Distance restraints & \\
Total NOE & 105 \\
Intraresidue & 65 \\
Inter-residue & 40 \\
$\quad$ Sequential $(|i-j|=1)$ & 38 \\
$\quad$ Nonsequential $(|i-j|>1)$ & 2 \\
Hydrogen bonds & 19 \\
Total dihedral-angle restraints & 216 \\
$\quad$ Base pair & 0 \\
Sugar pucker & 52 \\
$\quad$ Backbone & 164 \\
Based on A-form geometry & 216 \\
Structure statistics & \\
Violations (mean \pm s.d.) & \\
Distance constraints $(\AA)$ & $0.0 \pm 0.0$ \\
Dihedral-angle constraints $\left({ }^{\circ}\right)$ & $5.5 \pm 1.4$ \\
Deviations from idealized geometry & \\
Bond lengths $(\AA)$ & $0.004 \pm 0.000$ \\
Bond angles $\left({ }^{\circ}\right)$ & $0.616 \pm 0.006$ \\
Impropers $\left({ }^{\circ}\right)$ & $0.342 \pm 0.007$ \\
Average pairwise r.m.s. deviation $(\AA)^{a}$ & \\
All RNA heavy & 0.0466 \\
All nucleotides & 0.0553 \\
\hline & \\
&
\end{tabular}

a Pairwise r.m.s. deviation was calculated among ten refined structures. 


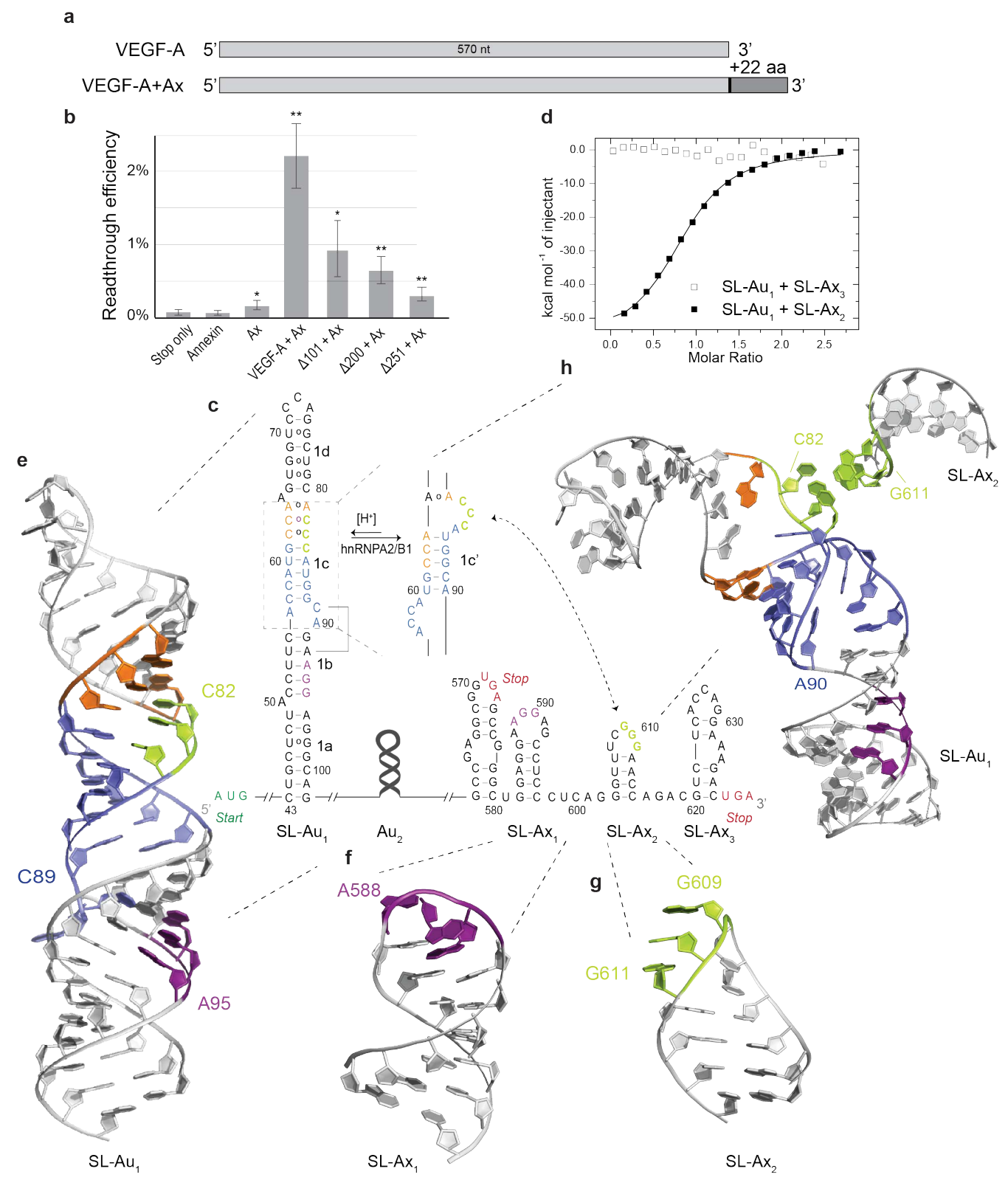
Functional readthrough levels show that only the full-length VEGF-A + Ax mRNA leads to substantial readthrough levels. Error bars indicate standard error $(n=3)$ and statistical tests were performed using a 2tailed t-test $(* \mathrm{p}<0.05, * * \mathrm{p}<0.005)$. (c) The secondary structure reveals an extended stem-loop for SL$\mathrm{Au}_{\mathrm{i}}$, including an alternate conformation (bases shown in orange and blue for simplicity), as well as shorter stem-loops SL-Ax $x_{1 \cdot 3}$ within the Ax-element. hnRNP binding sites are indicated in purple, stop codons are shown in red, and bases involved in the long-range interaction are shown in green. (d) ITC data show that $\mathrm{SL}-\mathrm{Au}_{1}$ is able to specifically interact with $\mathrm{SL}-\mathrm{Ax}_{2}$ (black squares), but not with $\mathrm{SL}-\mathrm{Ax}_{3}$

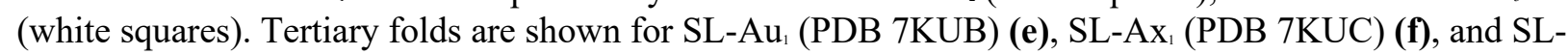
$\mathrm{Ax}_{2}$ (PDB 7KUD) (g) with the same base coloring as in (c). (h) A model was created showing the docking interaction between $\mathrm{SL}-\mathrm{Au}_{1}$ and $\mathrm{SL}-\mathrm{Ax}_{2}$. 

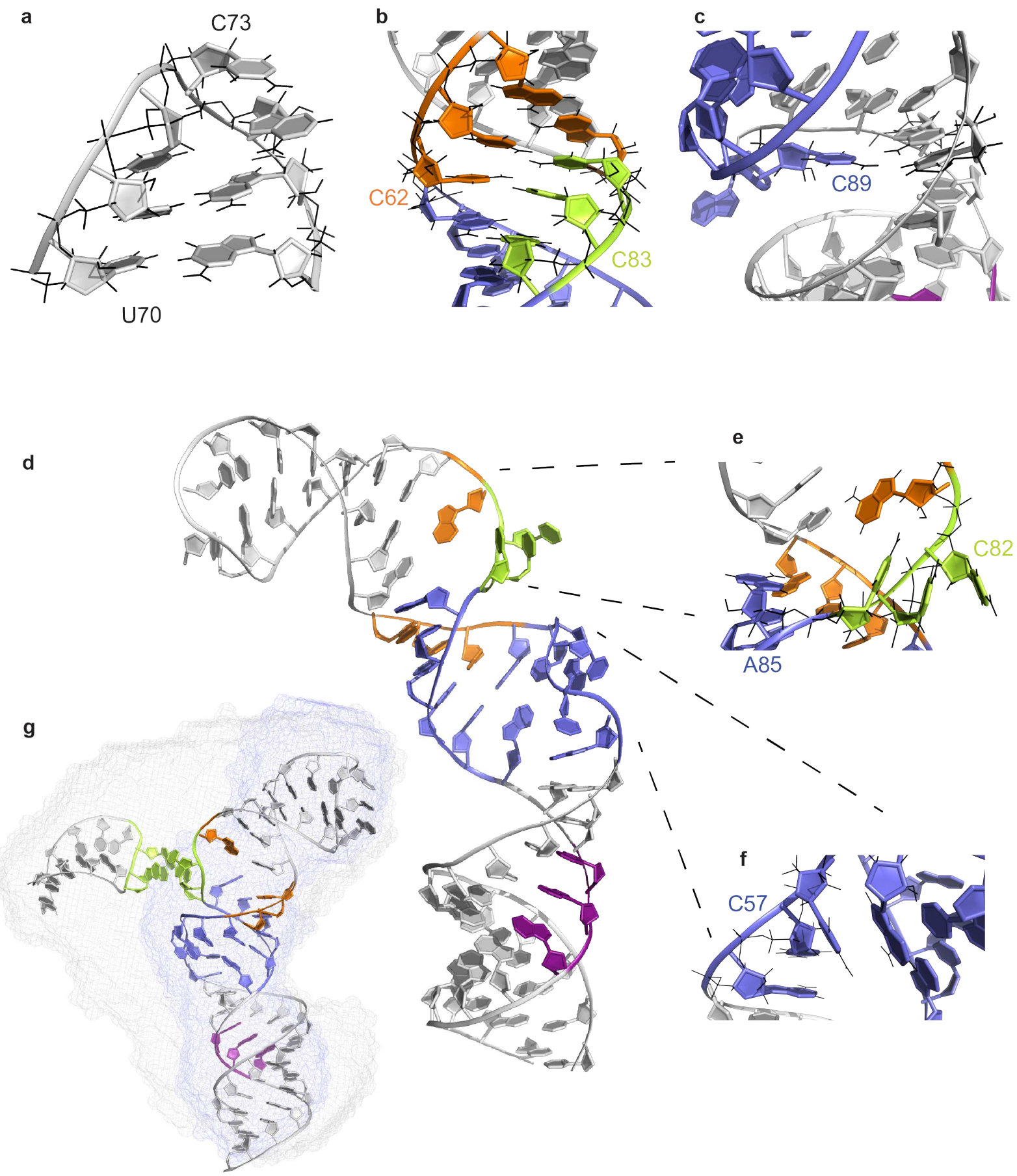

Figure 2: Structural features of SL-Au $u_{1}$. (a) Molecular detail of the $\mathrm{C}_{22} \mathrm{CA}$ triloop that caps SL-Au $u_{1 .}$ (b) Helix $1 \mathrm{c}$ of SL-Au $u_{1}$ contains 2 tandem C-C non-canonical basepairs. (c) SL-Au is able to form a triple base-pair interaction between $\mathrm{C}_{89}$ and the $\mathrm{A}_{92}-\mathrm{U}_{54}$ base-pair. (d) Helix 1c' assumes a kinked conformation and is flanked by an $\mathrm{A}_{56} \mathrm{CCA}$ bulge at the junction with helix $1 \mathrm{~b}$ (e) and an $\mathrm{A}_{81} \mathrm{CCCA}$ bulge at the junction with helix $1 \mathrm{~d}$ (f). (g) The density, as determined by SAXS, of free SL-Au in the 1c' conformation (blue) shows the bend described above. It also overlays with a model envelope (grey) of the $\mathrm{SL}-\mathrm{Au}_{1}: \mathrm{SL}-\mathrm{Ax}_{2}$ long-range interaction complex. Base coloring is as in Figure 1. 


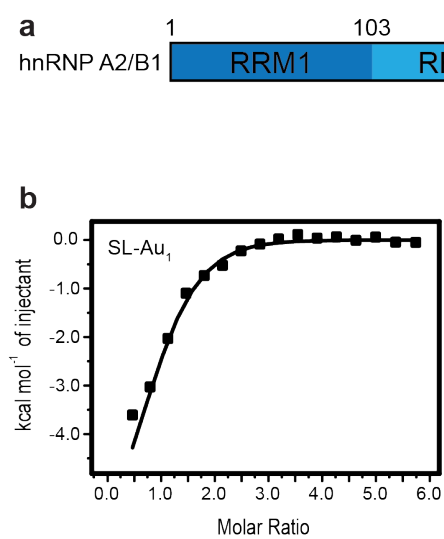

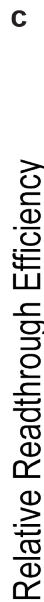
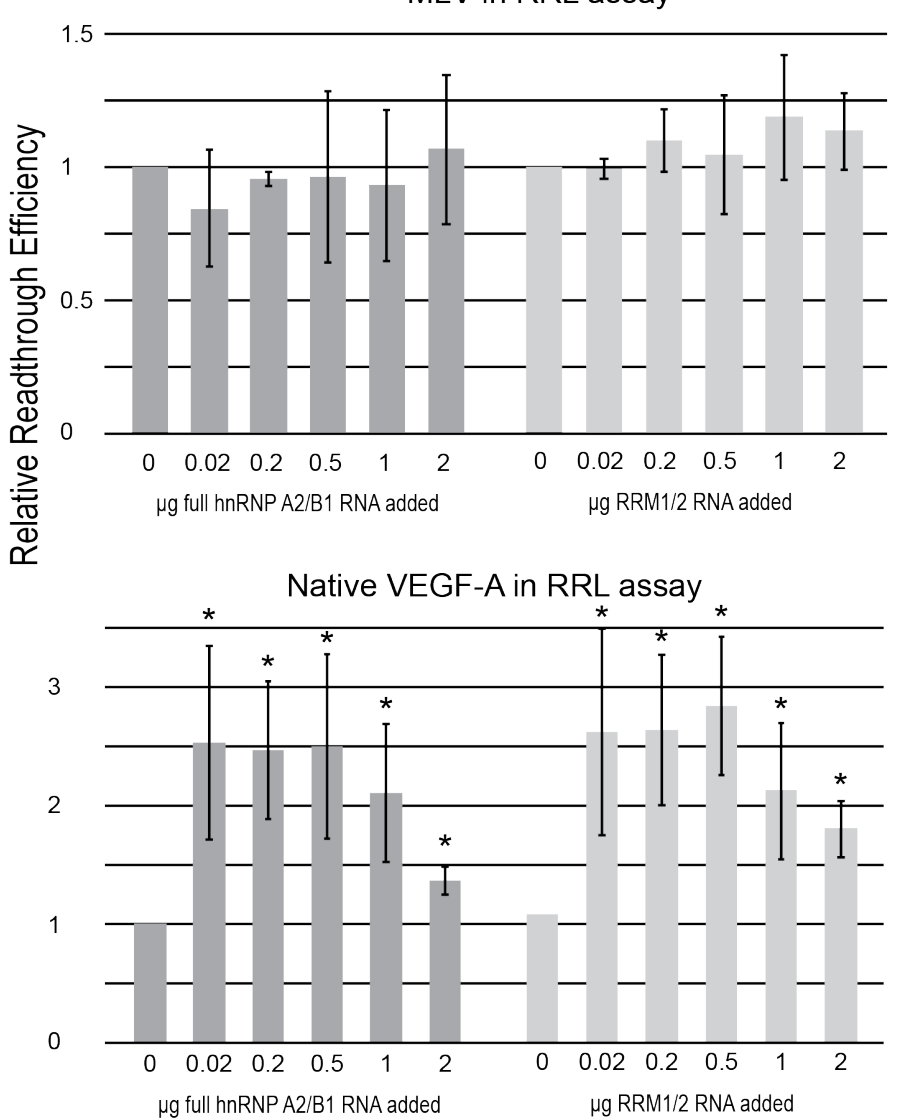

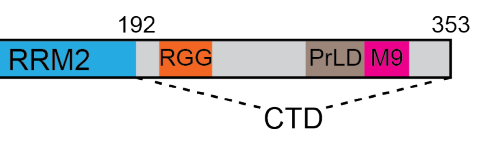

d

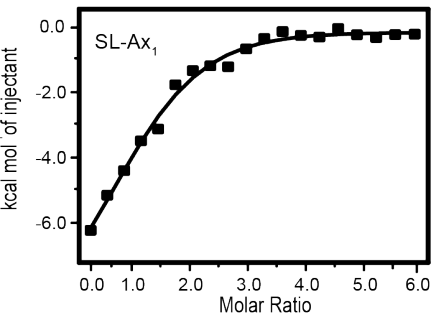

MLV in RRL assay
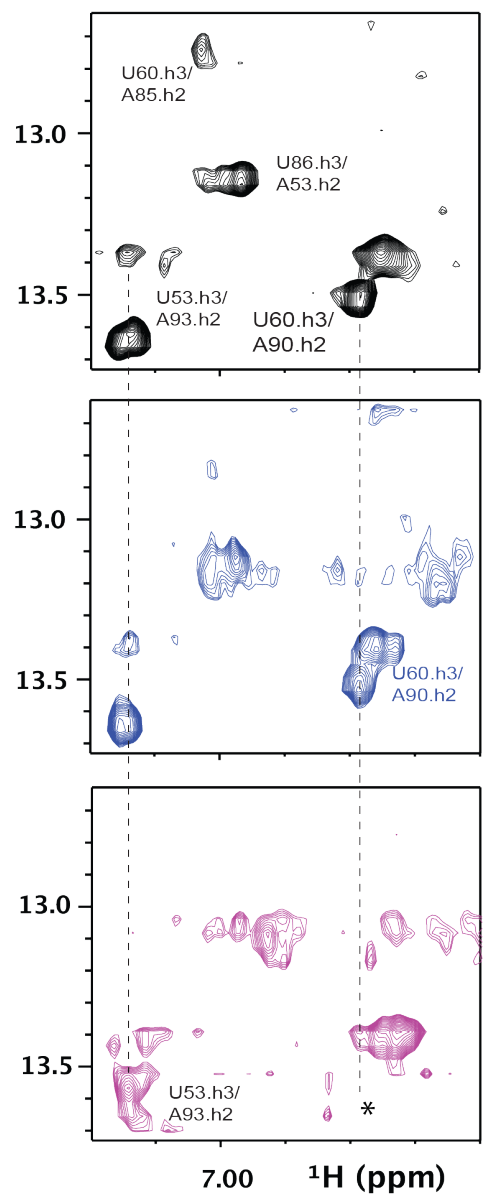

e

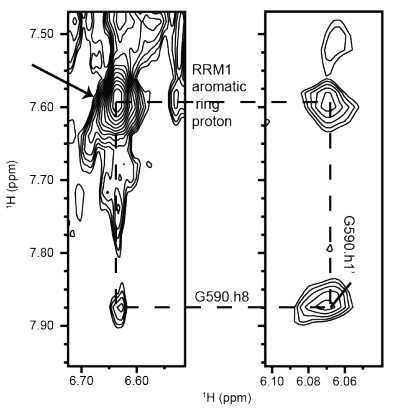

Figure 3: hnRNP A2/B1 binds to VEGF-A mRNA to help mediate readthrough. (a) Schematic of the hnRNP A2/B1 domain architecture showing its RNA-recognition motifs (RRM1 and RRM2) and Cterminal domain (CTD), consisting of an Arg-Gly-Gly box (RGG), prion-like domain (PrLD), and M9 transport sequence. (b) ITC binding studies of RRM1/2 to SL-Au (left) or SL-Ax $_{1}$ (right) show tight and specific binding. Representative curves of 2 trials are shown. (c) Rabbit reticulocyte lysate assays to which either full length hnRNP A2/B1 (dark grey) mRNA or RRM1/2 (light grey) was added show no difference in readthrough activity for an MLV control construct (top) or a VEGF-A construct (bottom). Error bars indicate standard error $(\mathrm{n}=3)$ and statistical tests were performed using a 2-tailed t-test $(* \mathrm{p}<$ 0.05). (d) Titration of RRM1/2 (black, 0 molar equivalents; blue, 0.3 molar equivalents; pink, 1 molar equivalent) into SL-Au $u_{1}$ at $280 \mathrm{~K}$ shows the shifting of several ${ }^{1} \mathrm{H}-{ }^{1} \mathrm{H}$ NMR peaks of base pairs disrupted by protein binding. (e) Titration of RRM1 into SL-Ax 1 at $311 \mathrm{~K}$ results in ${ }^{1} \mathrm{H}-{ }^{1} \mathrm{H}$ NOE cross-peaks between $\mathrm{G}_{590}$ and aromatic ring hydrogens of the protein. 


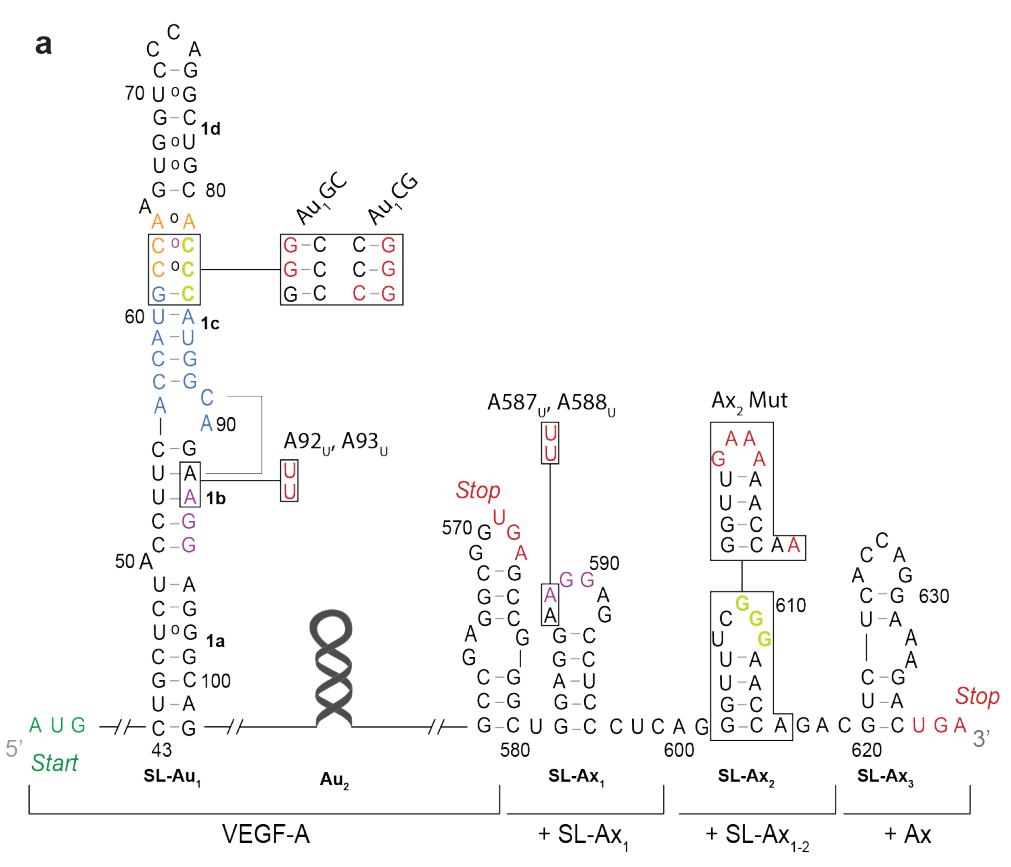

b
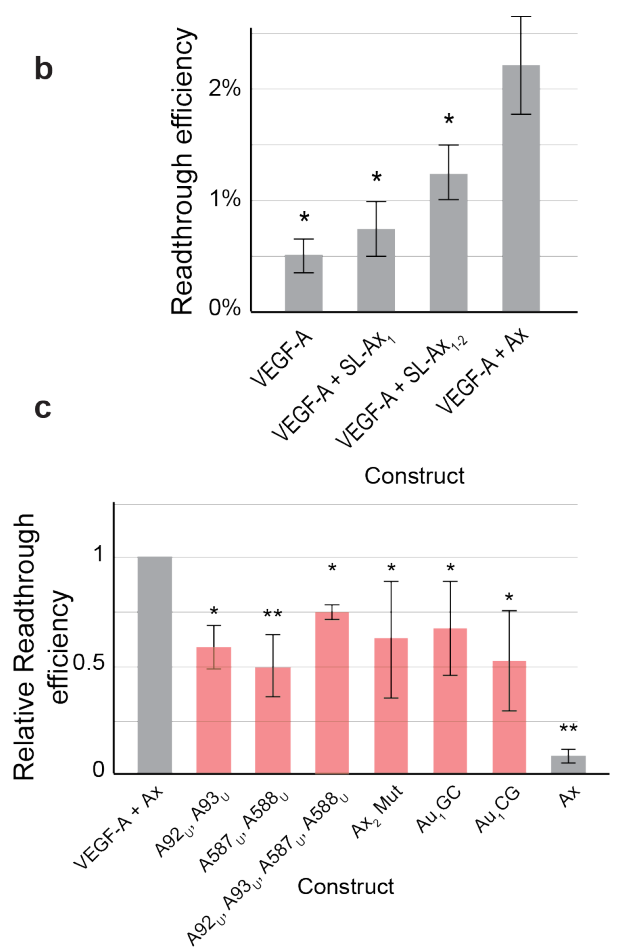

Figure 4: Each element of the VEGF-mRNA separately contributes toward readthrough activity. For truncated products, the maximal extent of the VEGF-A mRNA is indicated underneath the schematic. (b) Functional readthrough experiments show that each stem-loop within the Ax-element contributes toward readthrough. (c) Each tested mutation led to a decrease in readthrough levels, highlighting the importance of each region in mediating ribosomal readthrough. Error bars indicate standard error $(n=3)$ and statistical tests were performed using a 2-tailed t-test $(* \mathrm{p}<0.05, * * \mathrm{p}<0.005)$. 


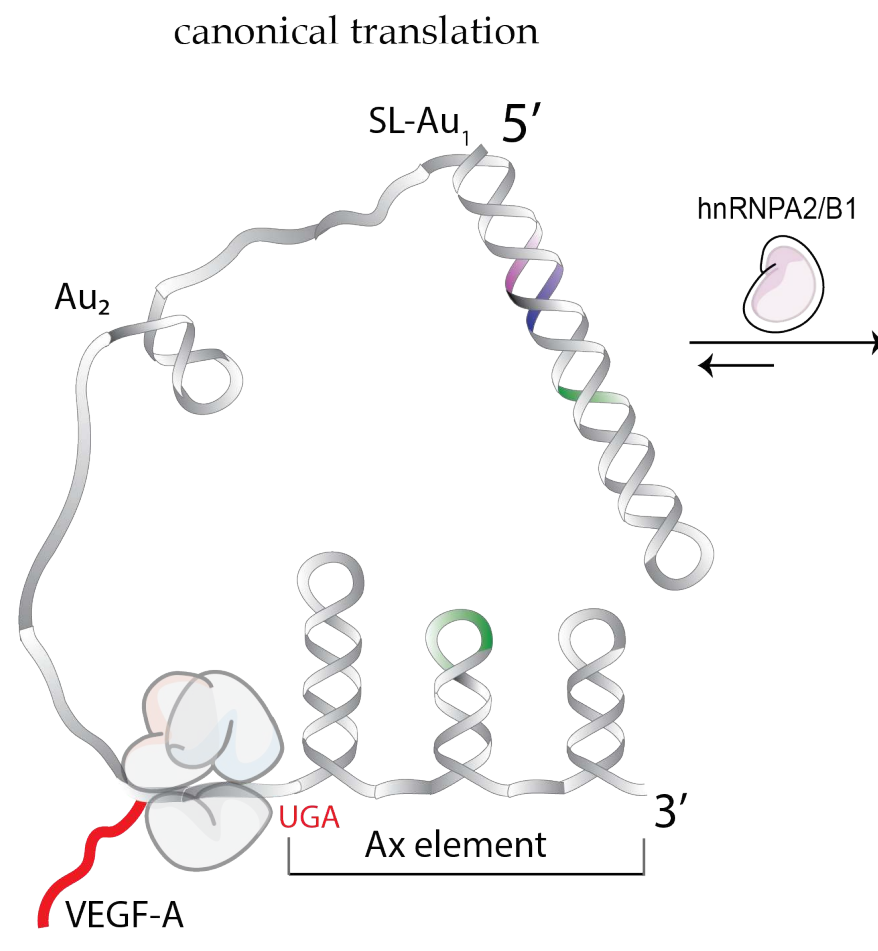

stop codon readthrough

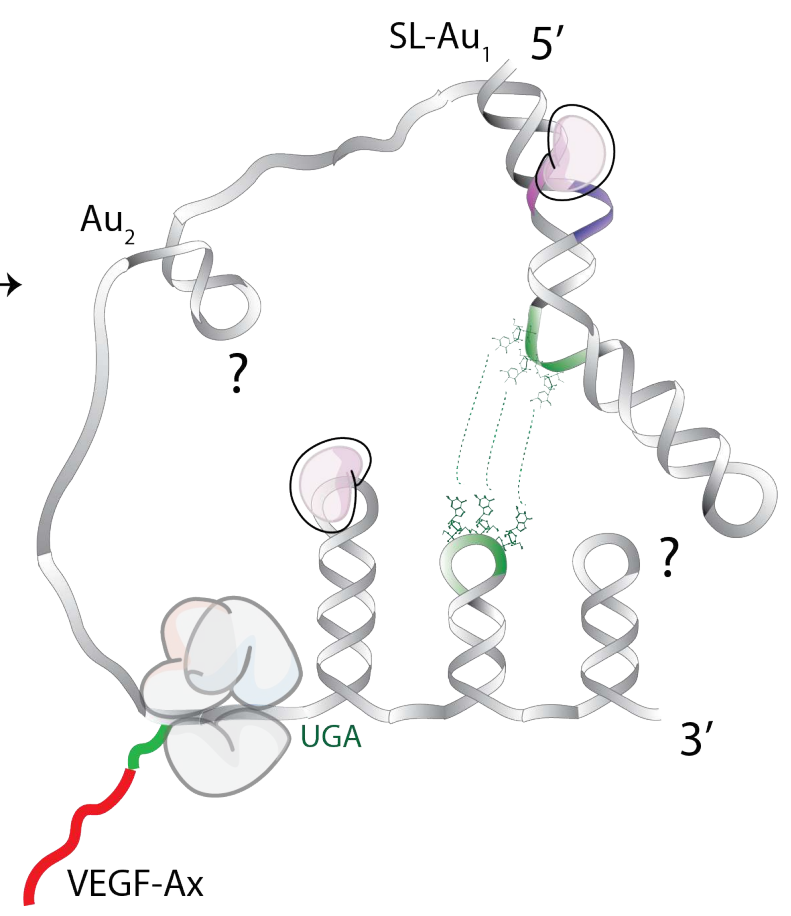

Figure 5: RNA alternate conformations mechanism of VEGF-A readthrough. A mechanistic model of the VEGF-A coding mRNA shows the formation of a linear SL-Au $\mathrm{Au}_{1}$ element at the 5 ' end of the RNA and three stem loops (SL-Ax $\left.{ }_{1-3}\right)$ at the 3' end of the RNA in the absence of hnRNP A2/B1. In the presence of hnRNP A2/B1, SL-Au 1 undergoes a register shift, exposing three cytosine residues. These three 561 cytosine residues are able to partake in long-range Watson-Crick base pairing with $\mathrm{SL}-\mathrm{Ax}_{2}$, thereby promoting translational stop-codon readthrough. 
a)

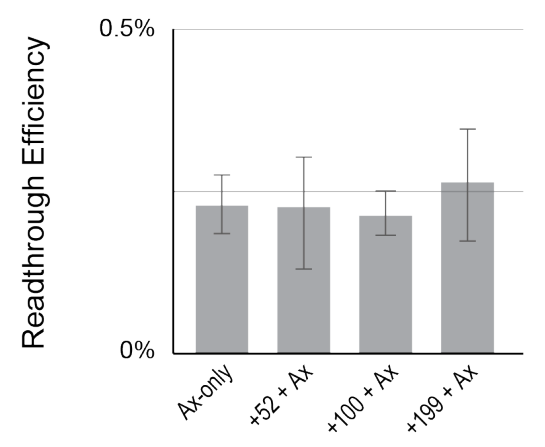

Construct b) 570

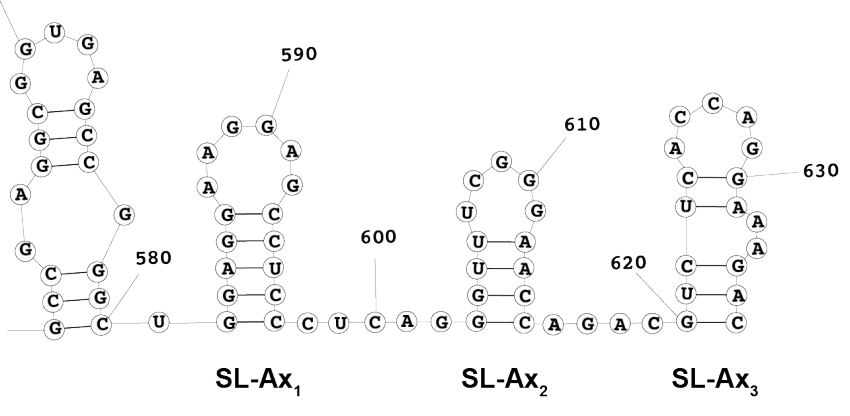

c)

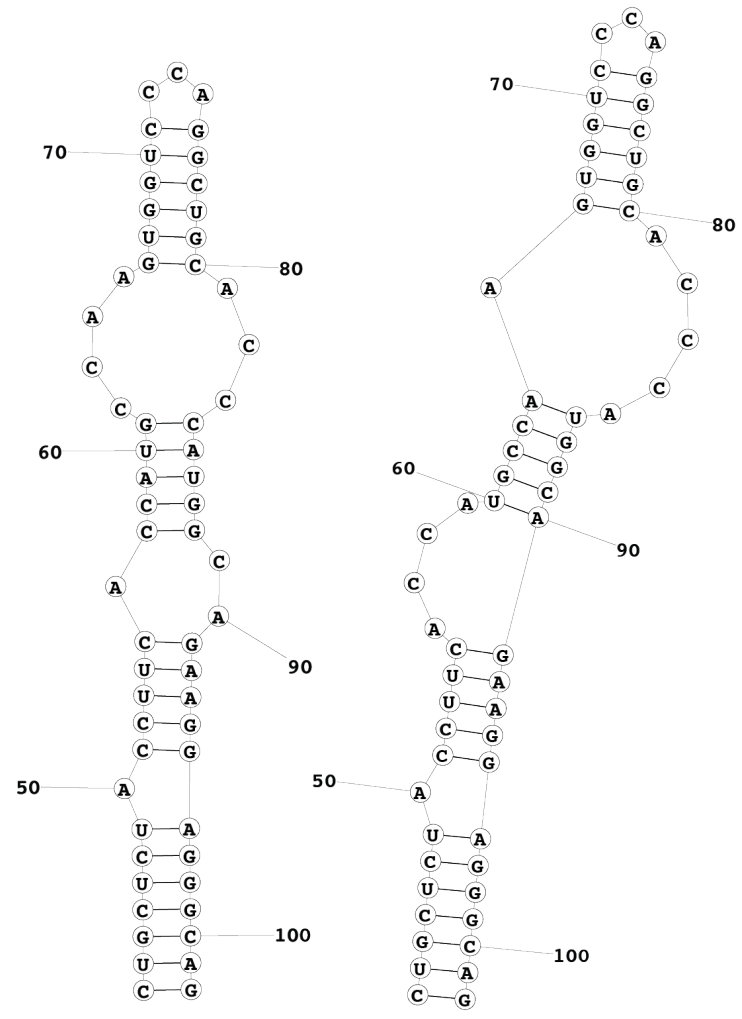

d)

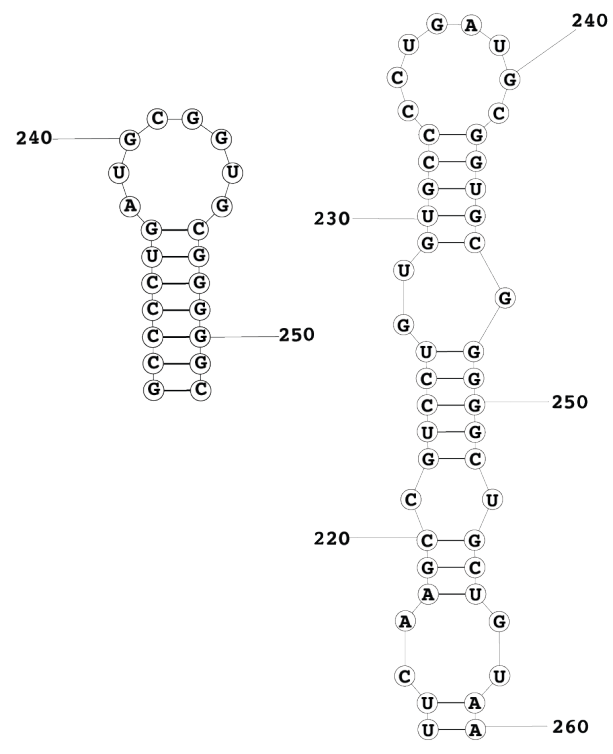

$\mathrm{Au}_{2}$

SL-Au,

Extended Data Figure 1: VEGF-A readthrough does not rely on local enhancers and DMS reactivities of the readthrough elements (a) Readthrough experiments indicate that the addition of sequences upstream of the Ax-element did not lead to increased levels in readthrough, ruling out the potential effects of local enhancers. Error bars indicate standard error $(n=3)$. DMS-MaPseq data show the predicted secondary structures of the Ax-element (b), the alternate conformations of SL-Au $\mathbf{A u}_{1}(\mathbf{c})$, and the alternate conformations of the second upstream signal, $\mathrm{Au}_{2}(\mathbf{d})$. 


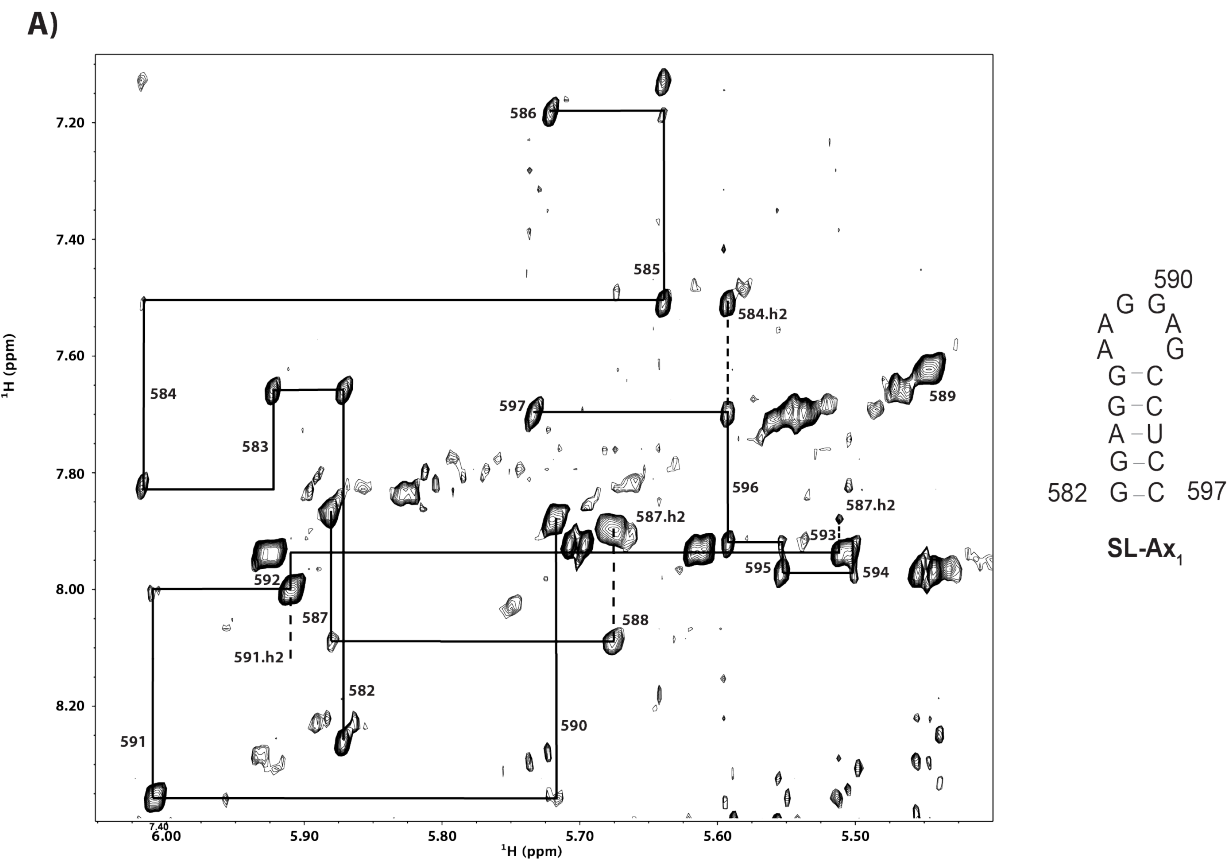

B)

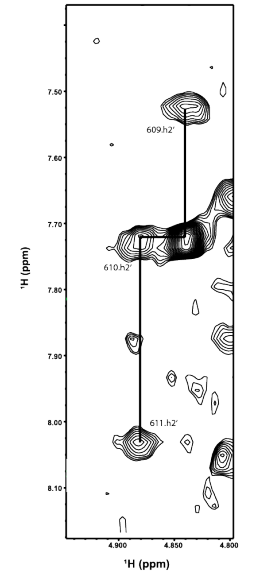

C)

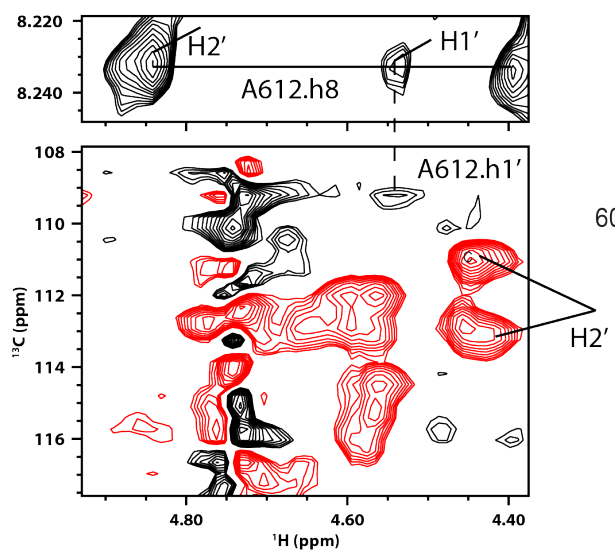

D)

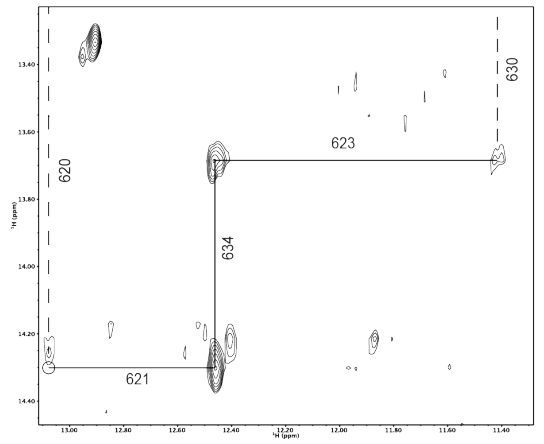

$C^{G}{ }^{610}$

$G$

$U-A$

G-C

$603 \mathrm{G}-\mathrm{C} 615$

$\mathrm{SL}-\mathrm{Ax}_{2}$

(a) $\mathrm{H}-1 \mathrm{H}$ NMR data at $311 \mathrm{~K}$ show that SL-Ax' assumes the fold predicted by DMS-MaPseq. (b) $\mathrm{H} H-1 \mathrm{H}$ NMR data $(311 \mathrm{~K})$ show that $\mathrm{G}_{609} \mathrm{GG}$ in SL-Ax 2 stack on top of each other and (c) HMQC data (311 K) show the upfield shift of the $A_{612} \mathrm{H} 1$ ' due to stacking on the ribose ring of $\mathrm{G}_{611}$. (d) Imino proton NMR assignments at $280 \mathrm{~K}$ for SL- $\mathrm{Ax}_{3}$ were obtained and confirm the secondary structure prediction and show 


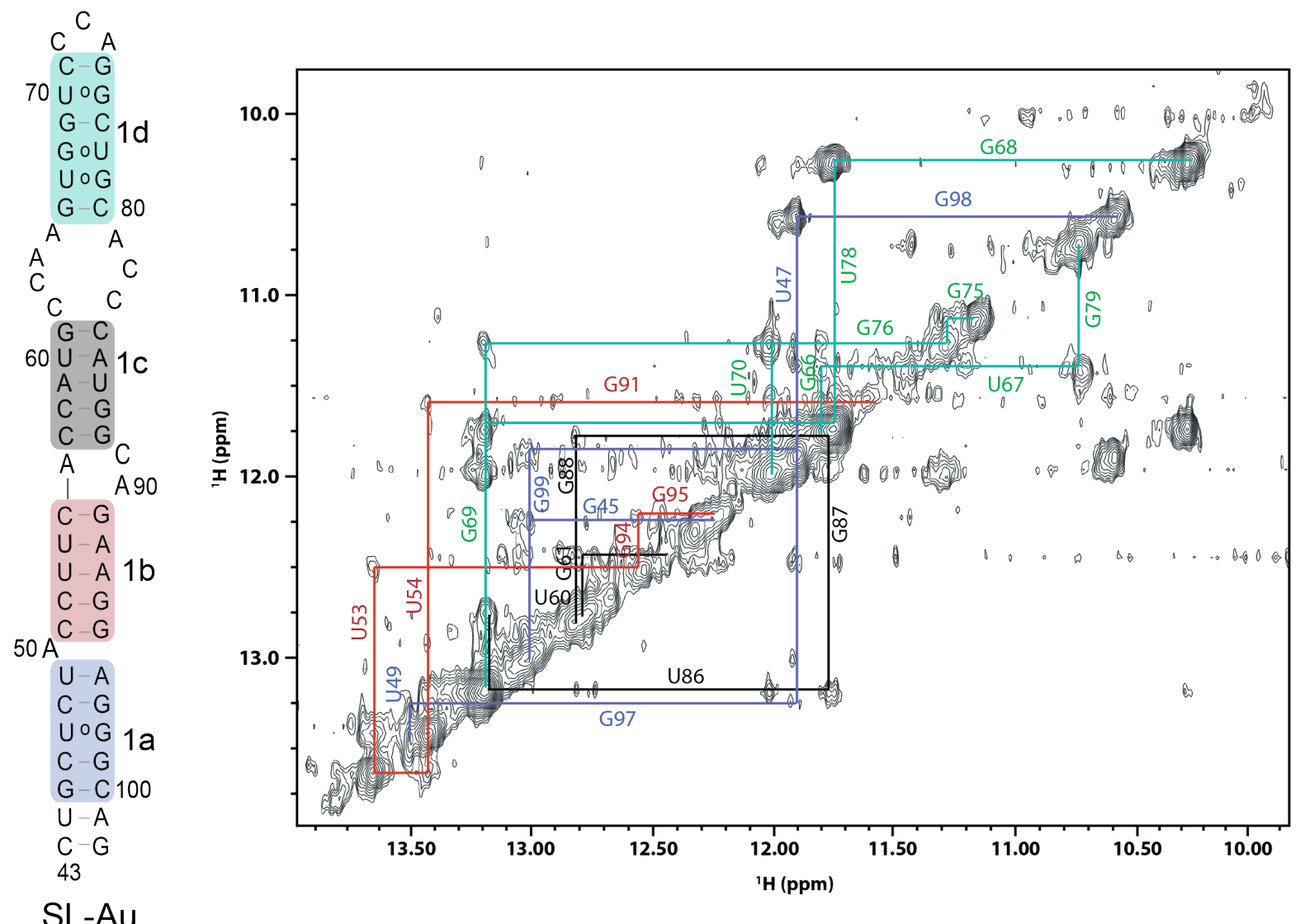

577 Extended Data Figure 3: NMR imino data confirm the predicted secondary structure of SL-Au ${ }_{1}$.

578 The complete imino proton NMR assignments $(280 \mathrm{~K})$ for SL-Au in the 1c conformation were obtained. 579 Imino assignments are colored to match the schematic of the secondary structure, which was subdivided 580 into helices 1a (blue), 1b (red), 1c (grey), and 1d (green). 


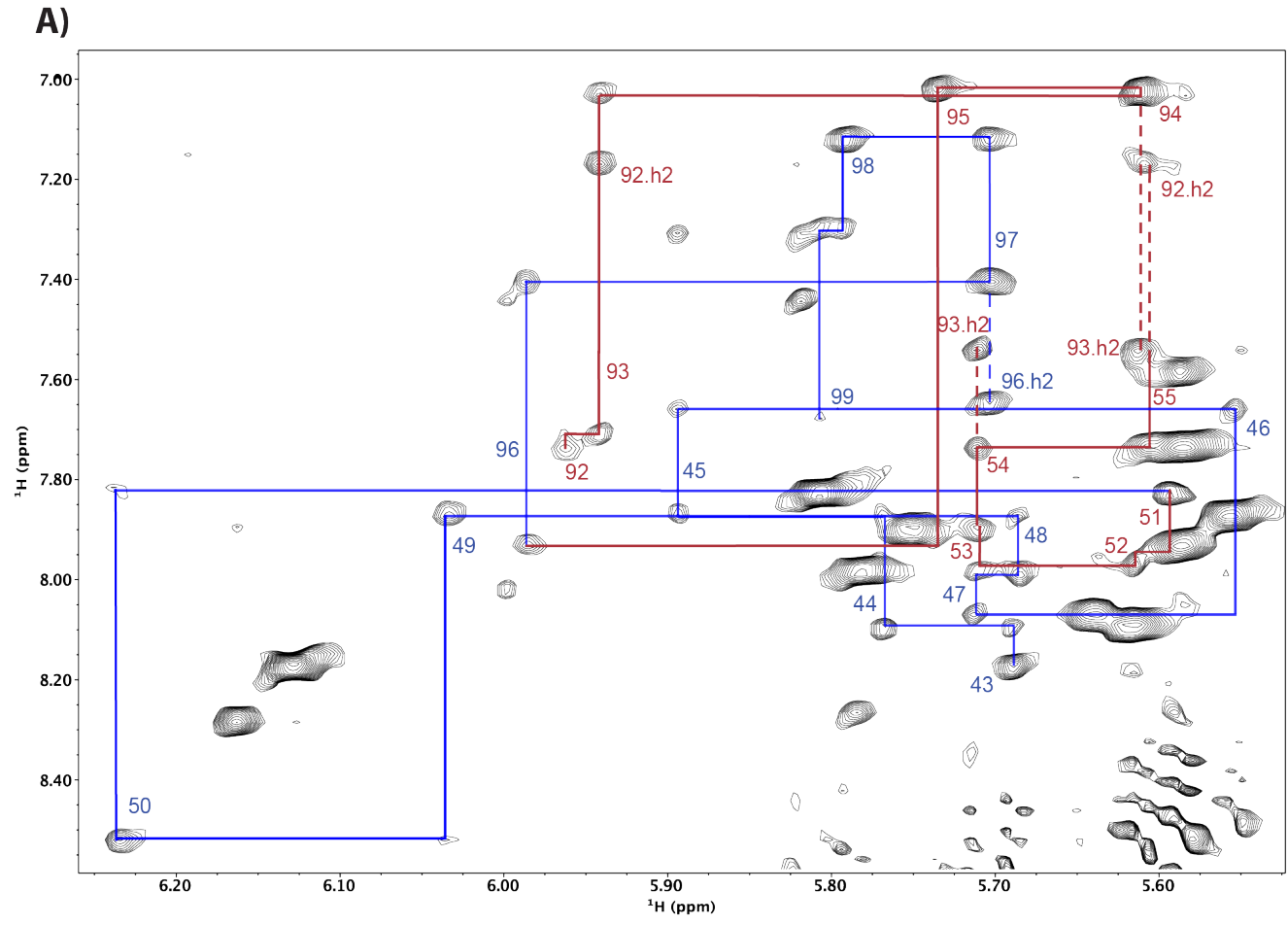

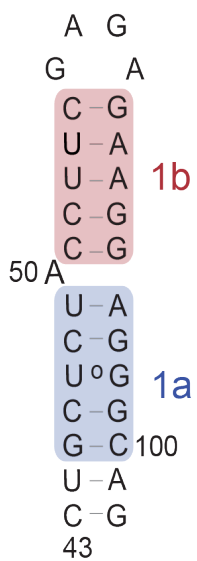

$1 a-1 b$

B)

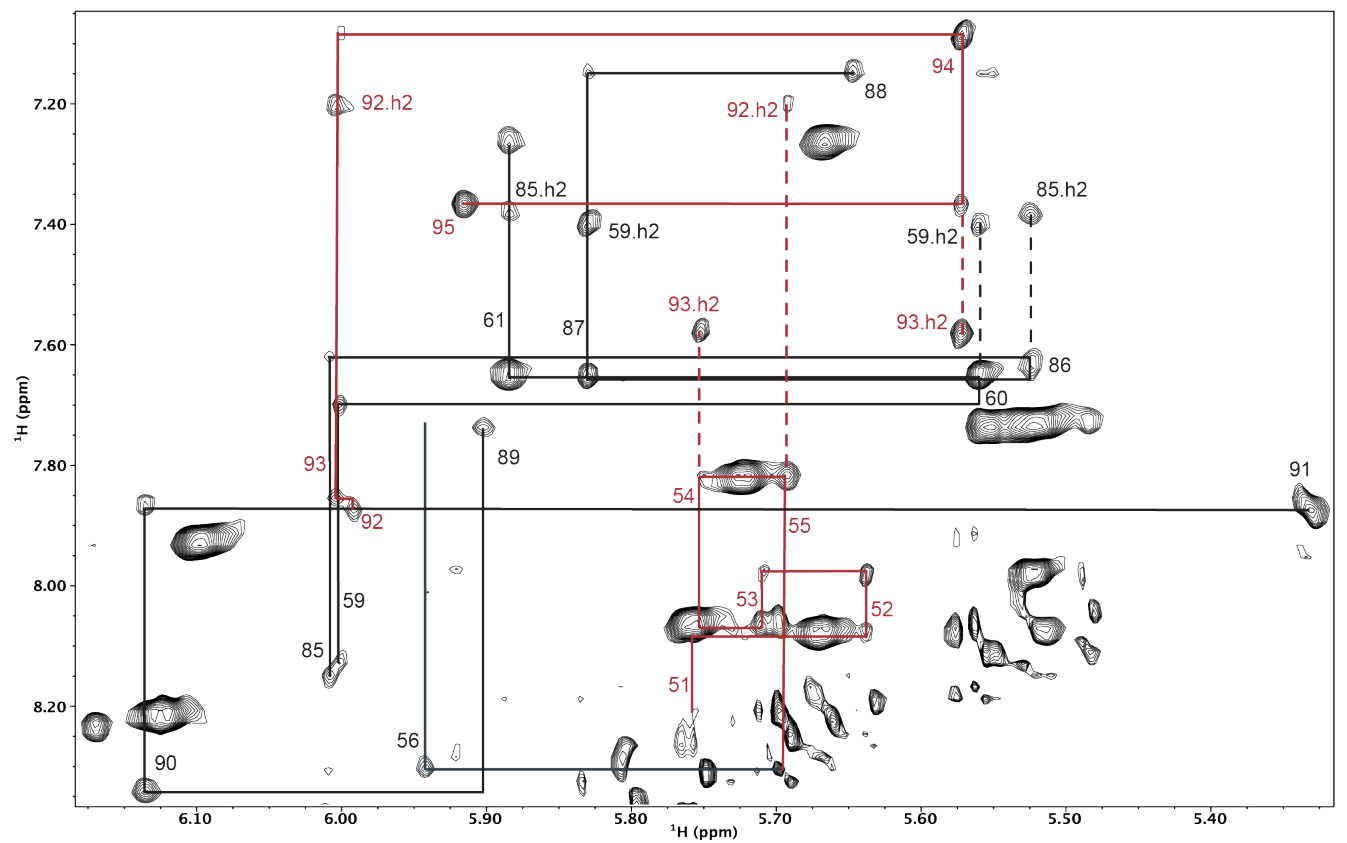

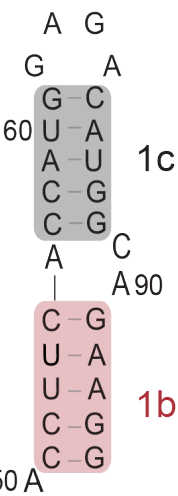

$1 b-1 c$
Extended Data Figure 4: NMR data confirm the predicted structures of SL-Au helices. Shortened RNA constructs were used to ascertain the structure of the full SL-Au, element. ${ }^{H} \mathrm{H}-1 \mathrm{H}$ NMR data $(311 \mathrm{~K})$ were collected to assign the peaks for helices (a) $1 \mathrm{a}-1 \mathrm{~b}$ and (b) $1 \mathrm{~b}-1 \mathrm{c}$. 
A)

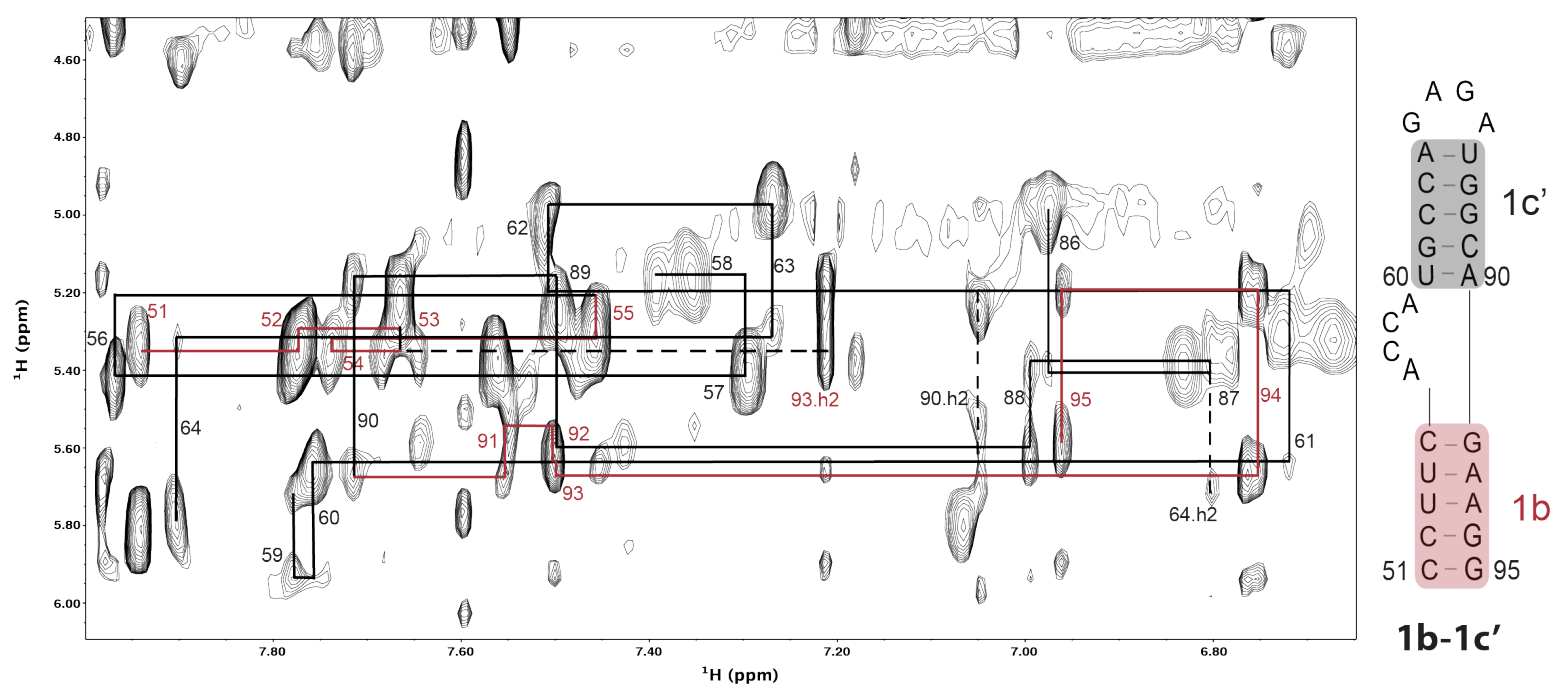

B)

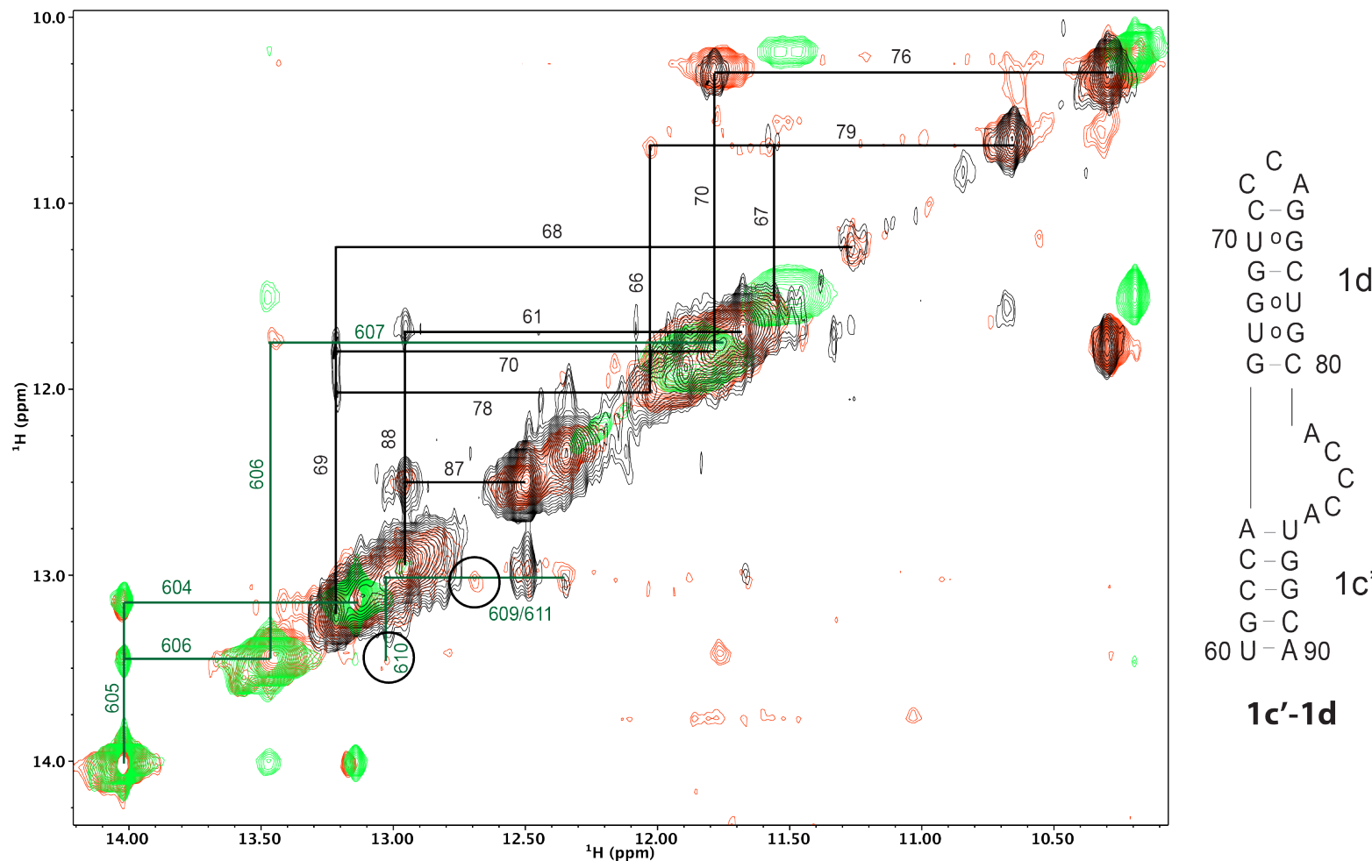

Extended Data Figure 5: NMR data reveal the existence of alternate structures of SL-Au helices that allow for long-range interactions. (a) $\mathrm{H}-\mathrm{-} \mathrm{H}$ NMR data $(311 \mathrm{~K})$ were collected to assign the peaks for the alternate conformation of helix 1c using a shortened helices 1b-1c' construct. (b) Imino proton NMR assignments $(298 \mathrm{~K})$ for helices $1 c^{\prime}-1 \mathrm{~d}$ (black) along with overlays of free SL-Ax (green) and the SL-Ax 2 : 1c'-1d complex (red) shows the formation of long-range basepairing involving $\mathrm{G}_{609} \mathrm{GG}$ of SL-Ax 2 . 
A)
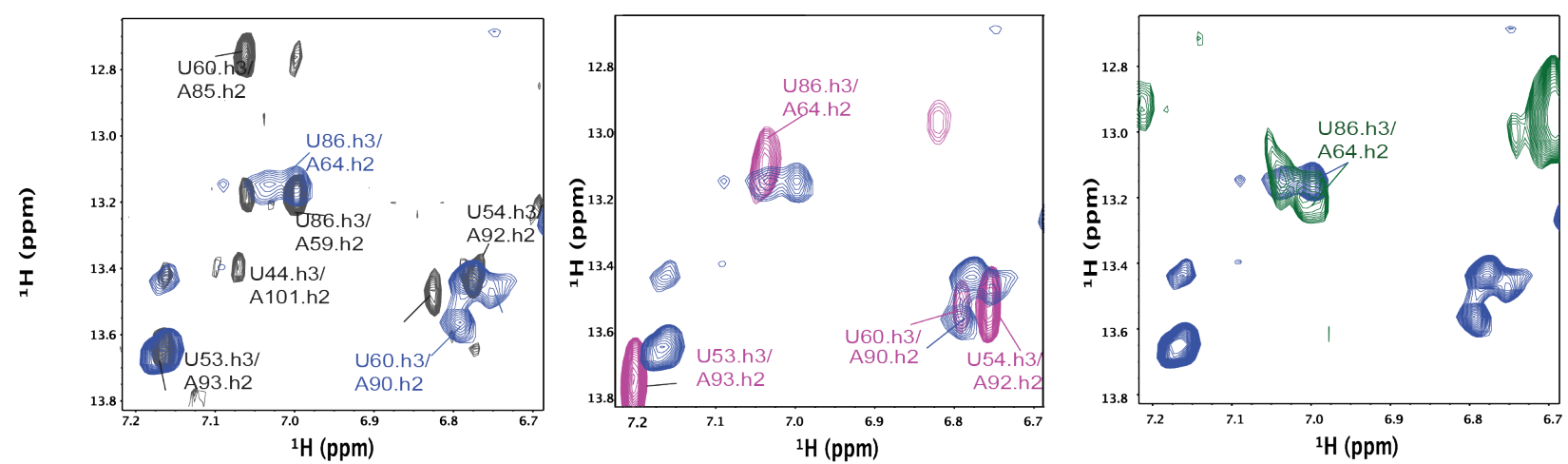

$\mathrm{Au}_{1}[15 \mathrm{mM} \mathrm{KCl}]$

$\mathrm{Au}_{1}[50 \mathrm{mM} \mathrm{KCl}]$

1b-1c'

$1 c^{\prime}-1 d$

B)

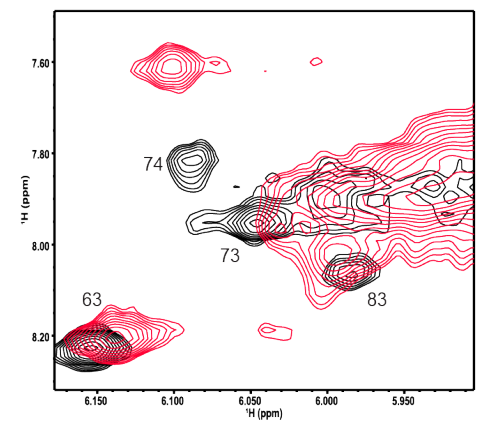

Extended Data Figure 6: NMR data confirm the presence of an equilibrium between helices 1c and 1c' of SL-Au $($ a) Multiple sets of signals are present in ' $\mathrm{H}-\mathrm{H} \mathrm{H}$ NMR data $(280 \mathrm{~K})$ at $15 \mathrm{mM} \mathrm{KCl}$ (black) and $50 \mathrm{mM} \mathrm{KCl}$ (blue), showing the salt dependence of the 1c to 1c' equilibrium. Overlays with shortened constructs 1b-1c' (purple) and 1c'-1d (green) show that the $50 \mathrm{mM} \mathrm{KCl}$ condition favors the $1 \mathrm{c}$ ' conformation. (b) The $1 \mathrm{c}$ to $1 \mathrm{c}$ ' equilibrium $(280 \mathrm{~K})$ is also $\mathrm{pH}$ dependent, showing distinct peaks at pH 5.6 (black) and pH 7.3 (red). 


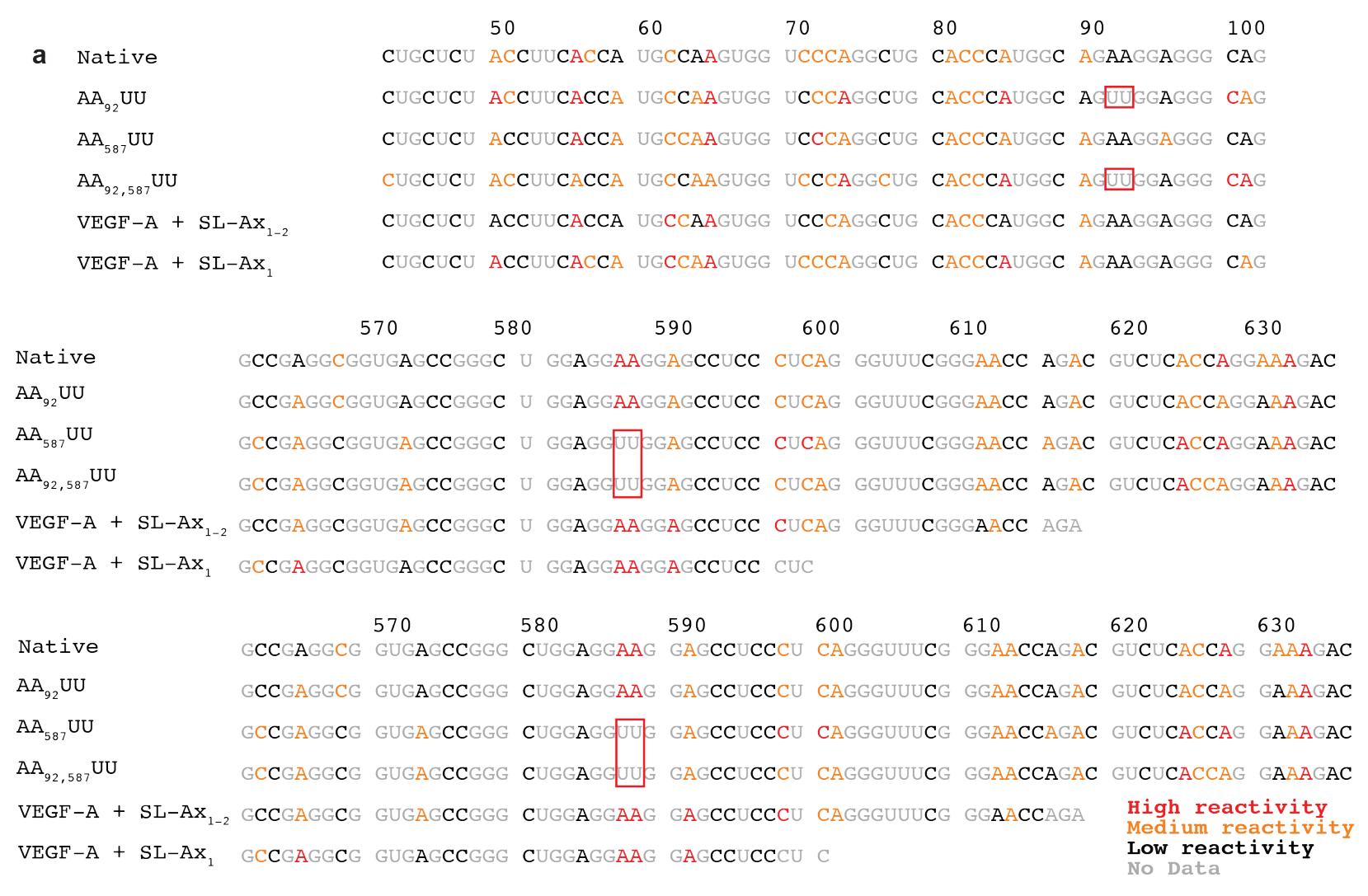
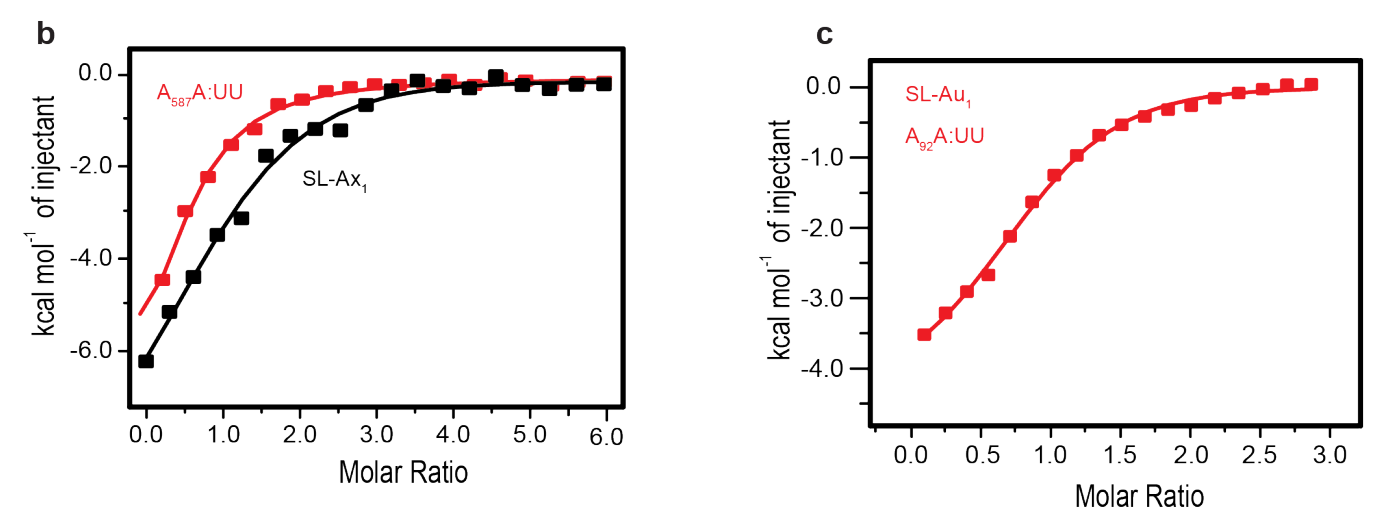

Extended Data Figure 7: Manipulations to the VEGF-A coding sequence maintain global mRNA structure and reduce $h n R N P A 2 / B 1$ affinity. (a) DMS reactivities for SL-Au $u_{1}$ and the Ax-element were collected for various VEGF-A mutant constructs to show there was no major changes in global fold. Mutations are indicated in red boxes while truncations are indicated by omitted nucleotides. $\mathrm{U}$ and $\mathrm{G}$ bases (grey) show no DMS reactivity while A and C bases may have low (black), medium (orange), or high (red) DMS reactivity. (b) ITC was used to determine a difference in binding affinity for RRM1/2 to native $S L-\mathrm{Ax}_{1}$ and $\mathrm{SL}-\mathrm{Ax}_{1}$ with an $\mathrm{A}_{58} \mathrm{~A}$ :UU mutation. To obtain a monophasic binding curve for the mutant, a phosphate buffer was used for this comparison. (c) An ITC binding curve was collected for $\mathrm{RRM} 1 / 2$ binding to $\mathrm{SL}-\mathrm{Au}_{1}$ with an $\mathrm{A}_{9_{2}} \mathrm{~A}$ :UU mutation in the same HEPES buffer as in Figure $3 \mathrm{~b}$. For these ITC experiments, representative curves of 3 trials are shown. 
610 This work was supported by HHMI grant 55108516 (to V.M.D'S.) and NSF-GRFP grant 1144152

611 (to N.O.W.). SAXS data was collected at the Advanced Light Source (ALS), SIBYLS beamline on

612 behalf of US DOE-BER, through the Integrated Diffraction Analysis Technologies (IDAT)

613 program. Additional support comes from the NIGMS project ALS-ENABLE (P30 GM124169) and

614 a High-End Instrumentation Grant S10OD018483.

615

\section{Accession codes}

617 Atomic coordinates have been deposited in the Protein Data Bank under accession codes PDB 618 7KUB (SL-Au1), PDB 7KUC (SL-Ax1), and PDB 7KUD (SL-Ax2). Chemical shifts have been 619 deposited in the Biological Magnetic Resonance Data Bank under accession codes 30816 (SL-Au1), 62030817 (SL-Ax1), and 30818 (SL-Ax2).

621

\section{Author contributions}

623 N.O.W., J.M.E., and V.M.D'S. conceived and designed the experiments. N.O.W. performed in624 cellulo and in-vitro functional assays. N.O.W. and J.M.E. purified the samples and performed the 625 NMR, ITC, and SAXS experiments. J.A. performed NMR experiments on SL-Ax1. N.O.W., J.M.E., 626 J.A. and V.M.D'S. performed the structural analyses. P.G., H.S., and S.R. performed the DMS627 MaPseq experiments and analysis. N.W., J.M.E., and V.M.D'S. interpreted the data and wrote the 628 manuscript.

629

630 Competing financial interests

631 The authors declare no competing financial interests. 
632 1. Schueren, F. \& Thoms, S. Functional Translational Readthrough: A Systems Biology Perspective. PLoS Genet 12, e1006196 (2016).

2. Dunn, J.G., Foo, C.K., Belletier, N.G., Gavis, E.R. \& Weissman, J.S. Ribosome profiling
reveals pervasive and regulated stop codon readthrough in Drosophila melanogaster. Elife 2, e01179 (2013).

3. Schueren, F. et al. Peroxisomal lactate dehydrogenase is generated by translational readthrough in mammals. Elife 3, e03640 (2014).

4. Stiebler, A.C. et al. Ribosomal readthrough at a short UGA stop codon context triggers dual localization of metabolic enzymes in Fungi and animals. PLoS Genet 10, e1004685 (2014).

5. Loughran, G. et al. Stop codon readthrough generates a C-terminally extended variant of the human vitamin D receptor with reduced calcitriol response. J Biol Chem 293, 44344444 (2018).

6. Brierley, I. et al. An efficient ribosomal frame-shifting signal in the polymerase-encoding region of the coronavirus IBV. EMBO J 6, 3779-85 (1987).

7. Yoshinaka, Y., Katoh, I., Copeland, T.D. \& Oroszlan, S. Murine leukemia virus protease is encoded by the gag-pol gene and is synthesized through suppression of an amber termination codon. Proc Natl Acad Sci U S A 82, 1618-22 (1985).

8. Steneberg, P. \& Samakovlis, C. A novel stop codon readthrough mechanism produces functional Headcase protein in Drosophila trachea. EMBO Rep 2, 593-7 (2001).

9. Curran, J.F. \& Yarus, M. Use of tRNA suppressors to probe regulation of Escherichia coli release factor 2. J Mol Biol 203, 75-83 (1988).

10. Jungreis, I. et al. Evolutionary Dynamics of Abundant Stop Codon Readthrough. Mol Biol Evol 33, 3108-3132 (2016).

11. Eswarappa, S.M. et al. Programmed translational readthrough generates antiangiogenic VEGF-Ax. Cell 157, 1605-18 (2014).

12. Xin, H., Zhong, C., Nudleman, E. \& Ferrara, N. Evidence for Pro-angiogenic Functions of VEGF-Ax. Cell 167, 275-284 e6 (2016).

13. Dever, T.E., Dinman, J.D. \& Green, R. Translation Elongation and Recoding in Eukaryotes. Cold Spring Harb Perspect Biol 10(2018).

14. Gesteland, R.F. \& Atkins, J.F. Recoding: dynamic reprogramming of translation. Annu Rev Biochem 65, 741-68 (1996).

15. Bidou, L., Rousset, J.P. \& Namy, O. Translational errors: from yeast to new therapeutic targets. FEMS Yeast Res 10, 1070-82 (2010).

16. Firth, A.E. \& Brierley, I. Non-canonical translation in RNA viruses. J Gen Virol 93, 1385409 (2012).

17. Namy, O., Moran, S.J., Stuart, D.I., Gilbert, R.J. \& Brierley, I. A mechanical explanation of RNA pseudoknot function in programmed ribosomal frameshifting. Nature 441, 244-7 (2006).

18. Barry, J.K. \& Miller, W.A. A -1 ribosomal frameshift element that requires base pairing across four kilobases suggests a mechanism of regulating ribosome and replicase traffic on a viral RNA. Proc Natl Acad Sci U S A 99, 11133-8 (2002). 
19. Cimino, P.A., Nicholson, B.L., Wu, B., Xu, W. \& White, K.A. Multifaceted regulation of translational readthrough by RNA replication elements in a tombusvirus. PLoS Pathog 7, e1002423 (2011).

20. Xu, Y. et al. A Stem-Loop Structure in Potato Leafroll Virus Open Reading Frame 5 (ORF5) Is Essential for Readthrough Translation of the Coat Protein ORF Stop Codon 700 Bases Upstream. J Virol 92(2018).

21. Napthine, S. et al. Protein-directed ribosomal frameshifting temporally regulates gene expression. Nat Commun 8, 15582 (2017).

22. Napthine, $\mathrm{S}$. et al. A novel role for poly(C) binding proteins in programmed ribosomal frameshifting. Nucleic Acids Res 44, 5491-503 (2016).

23. Leung, D.W., Cachianes, G., Kuang, W.J., Goeddel, D.V. \& Ferrara, N. Vascular endothelial growth factor is a secreted angiogenic mitogen. Science 246, 1306-9 (1989).

24. Melincovici, C.S. et al. Vascular endothelial growth factor (VEGF) - key factor in normal and pathological angiogenesis. Rom J Morphol Embryol 59, 455-467 (2018).

25. Loughran, G., Howard, M.T., Firth, A.E. \& Atkins, J.F. Avoidance of reporter assay distortions from fused dual reporters. RNA 23, 1285-1289 (2017).

26. Grentzmann, G., Ingram, J.A., Kelly, P.J., Gesteland, R.F. \& Atkins, J.F. A dual-luciferase reporter system for studying recoding signals. RNA 4, 479-86 (1998).

27. Cridge, A.G., Crowe-McAuliffe, C., Mathew, S.F. \& Tate, W.P. Eukaryotic translational termination efficiency is influenced by the $3^{\prime}$ nucleotides within the ribosomal mRNA channel. Nucleic Acids Res 46, 1927-1944 (2018).

28. Zubradt, M. et al. DMS-MaPseq for genome-wide or targeted RNA structure probing in vivo. Nat Methods 14, 75-82 (2017).

29. Vander Meulen, K.A., Davis, J.H., Foster, T.R., Record, M.T., Jr. \& Butcher, S.E. Thermodynamics and folding pathway of tetraloop receptor-mediated RNA helical packing. J Mol Biol 384, 702-17 (2008).

30. Hansen, J.L., Schmeing, T.M., Moore, P.B. \& Steitz, T.A. Structural insights into peptide bond formation. Proc Natl Acad Sci U S A 99, 11670-5 (2002).

31. Brown, J.A. Unraveling the structure and biological functions of RNA triple helices. Wiley Interdiscip Rev RNA 11, e1598 (2020).

32. Jain, N., Morgan, C.E., Rife, B.D., Salemi, M. \& Tolbert, B.S. Solution Structure of the HIV1 Intron Splicing Silencer and Its Interactions with the UP1 Domain of Heterogeneous Nuclear Ribonucleoprotein (hnRNP) A1. J Biol Chem 291, 2331-44 (2016).

33. Kooshapur, H. et al. Structural basis for terminal loop recognition and stimulation of primiRNA-18a processing by hnRNP A1. Nat Commun 9, 2479 (2018).

34. Morgan, C.E. et al. The First Crystal Structure of the UP1 Domain of hnRNP A1 Bound to RNA Reveals a New Look for an Old RNA Binding Protein. J Mol Biol 427, 3241-3257 (2015).

35. Tolbert, M. et al. HnRNP A1 Alters the Structure of a Conserved Enterovirus IRES Domain to Stimulate Viral Translation. J Mol Biol 429, 2841-2858 (2017).

36. Wu, B. et al. Molecular basis for the specific and multivariant recognitions of RNA substrates by human hnRNP A2/B1. Nat Commun 9, 420 (2018).

37. Dreyfuss, G., Matunis, M.J., Pinol-Roma, S. \& Burd, C.G. hnRNP proteins and the biogenesis of mRNA. Annu Rev Biochem 62, 289-321 (1993). 
38. Geuens, T., Bouhy, D. \& Timmerman, V. The hnRNP family: insights into their role in health and disease. Hum Genet 135, 851-67 (2016).

39. Han, S.P., Tang, Y.H. \& Smith, R. Functional diversity of the hnRNPs: past, present and perspectives. Biochem J 430, 379-92 (2010).

40. Kwak, H., Park, M.W. \& Jeong, S. Annexin A2 binds RNA and reduces the frameshifting efficiency of infectious bronchitis virus. PLoS One 6, e24067 (2011).

41. Gao, F. \& Simon, A.E. Multiple Cis-acting elements modulate programmed -1 ribosomal frameshifting in Pea enation mosaic virus. Nucleic Acids Res 44, 878-95 (2016).

42. Wu, M.T. \& D'Souza, V. Alternate RNA Structures. Cold Spring Harb Perspect Biol 12(2020).

43. Vicens, Q., Kieft, J.S. \& Rissland, O.S. Revisiting the Closed-Loop Model and the Nature of mRNA 5'-3' Communication. Mol Cell 72, 805-812 (2018).

44. Yoffe, A.M., Prinsen, P., Gelbart, W.M. \& Ben-Shaul, A. The ends of a large RNA molecule are necessarily close. Nucleic Acids Res 39, 292-9 (2011).

45. Lai, W.C. et al. mRNAs and IncRNAs intrinsically form secondary structures with short end-to-end distances. Nat Commun 9, 4328 (2018).

46. Adivarahan, S. et al. Spatial Organization of Single mRNPs at Different Stages of the Gene Expression Pathway. Mol Cell 72, 727-738 e5 (2018).

47. Khong, A. \& Parker, R. mRNP architecture in translating and stress conditions reveals an ordered pathway of mRNP compaction. J Cell Biol 217, 4124-4140 (2018).

48. Harper, S. \& Speicher, D.W. Purification of proteins fused to glutathione S-transferase. Methods Mol Biol 681, 259-80 (2011).

49. D'Souza, V., Dey, A., Habib, D. \& Summers, M.F. NMR structure of the 101-nucleotide core encapsidation signal of the Moloney murine leukemia virus. J Mol Biol 337, 427-42 (2004).

50. Norris, M., Fetler, B., Marchant, J. \& Johnson, B.A. NMRFx Processor: a cross-platform NMR data processing program. J Biomol NMR 65, 205-216 (2016).

51. Guntert, P. Automated NMR structure calculation with CYANA. Methods Mol Biol 278, 353-78 (2004).

52. Schwieters, C.D., Kuszewski, J.J., Tjandra, N. \& Clore, G.M. The Xplor-NIH NMR molecular structure determination package. J Magn Reson 160, 65-73 (2003).

53. Schwieters, C.D. \& Clore, G.M. Using small angle solution scattering data in Xplor-NIH structure calculations. Prog Nucl Magn Reson Spectrosc 80, 1-11 (2014).

54. Rouskin, S., Zubradt, M., Washietl, S., Kellis, M. \& Weissman, J.S. Genome-wide probing of RNA structure reveals active unfolding of mRNA structures in vivo. Nature 505, 701-5 (2014).

55. Dyer, K.N. et al. High-throughput SAXS for the characterization of biomolecules in solution: a practical approach. Methods Mol Biol 1091, 245-58 (2014).

56. Forster, S., Apostol, L. \& Bras, W. Scatter: software for the analysis of nano- and mesoscale small-angle scattering. Journal of Applied Crystallography 43, 639-646 (2010). 


\section{Figures}

a

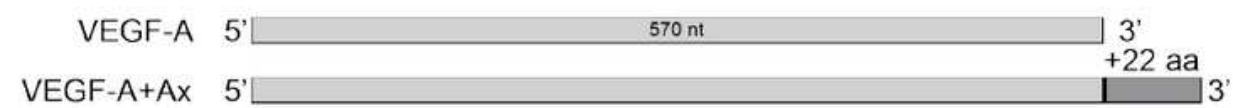

b
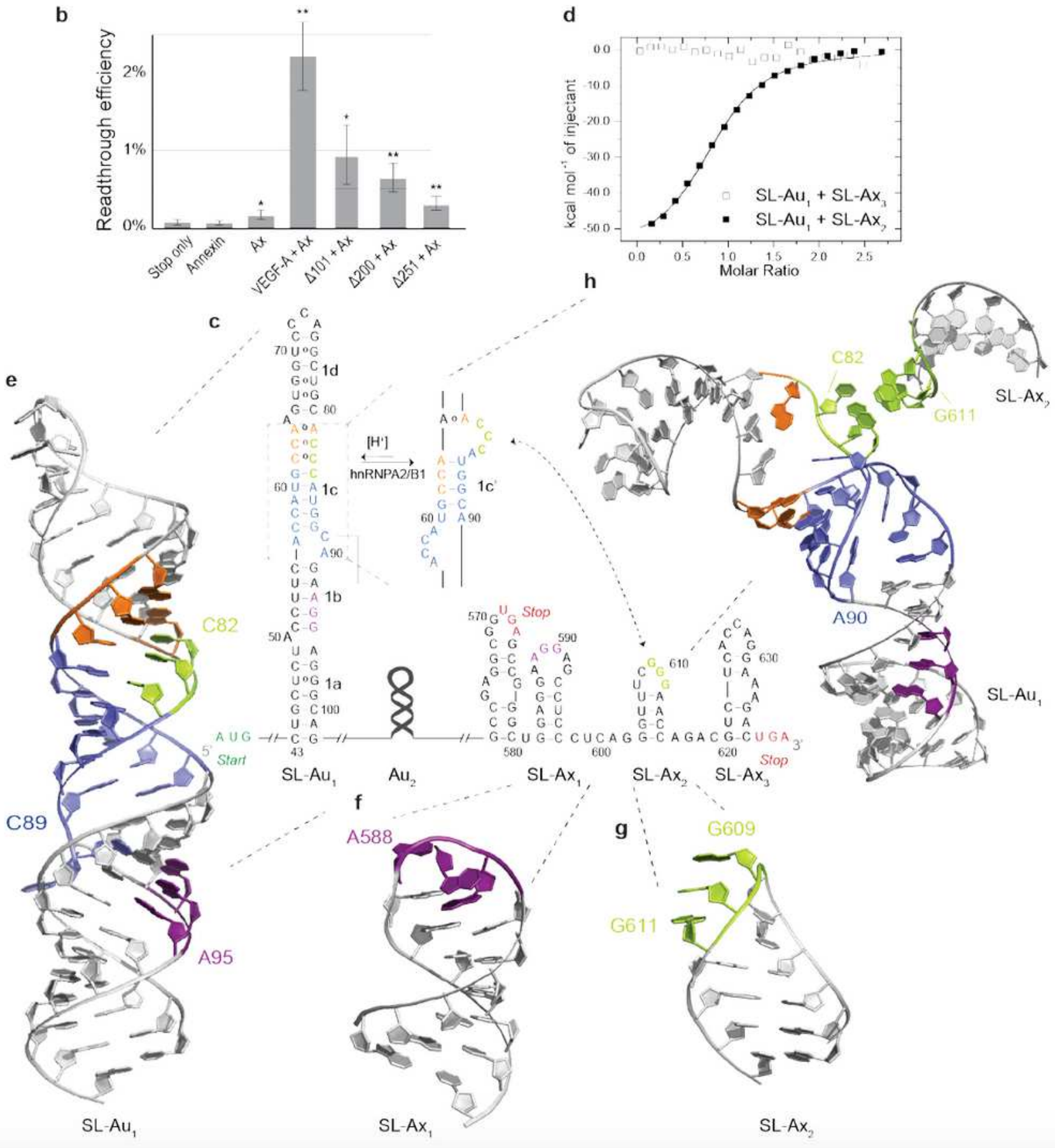

Figure 1

Identification and structures of the elements involved in mediating VEGF-A readthrough. (a) Schematic diagram of the VEGF-A mRNA without (top) and with (bottom) the Ax-element. (b) Functional readthrough levels show that only the full-length VEGF-A + Ax mRNA leads to substantial readthrough levels. Error 
bars indicate standard error $(n=3)$ and statistical tests were performed using a 2- tailed t-test $\left({ }^{*} p<0.05\right.$, $\star \star p<0.005$ ). (c) The secondary structure reveals an extended stem-loop for SLAu 1 , including an alternate conformation (bases shown in orange and blue for simplicity), as well as shorter stem-loops SLAx1-3 within the Ax-element. hnRNP binding sites are indicated in purple, stop codons are shown in red, and bases involved in the long-range interaction are shown in green. (d) ITC data show that SL-Au1 is able to specifically interact with SL-Ax2 (black squares), but not with SL-Ax3 (white squares). Tertiary folds are shown for SL-Au1 (PDB 7KUB) (e), SL-Ax1 (PDB 7KUC) (f), and SLAx 2 (PDB 7KUD) (g) with the same base coloring as in (c). (h) A model was created showing the docking interaction between SL-Au1 and SL-Ax2 . 

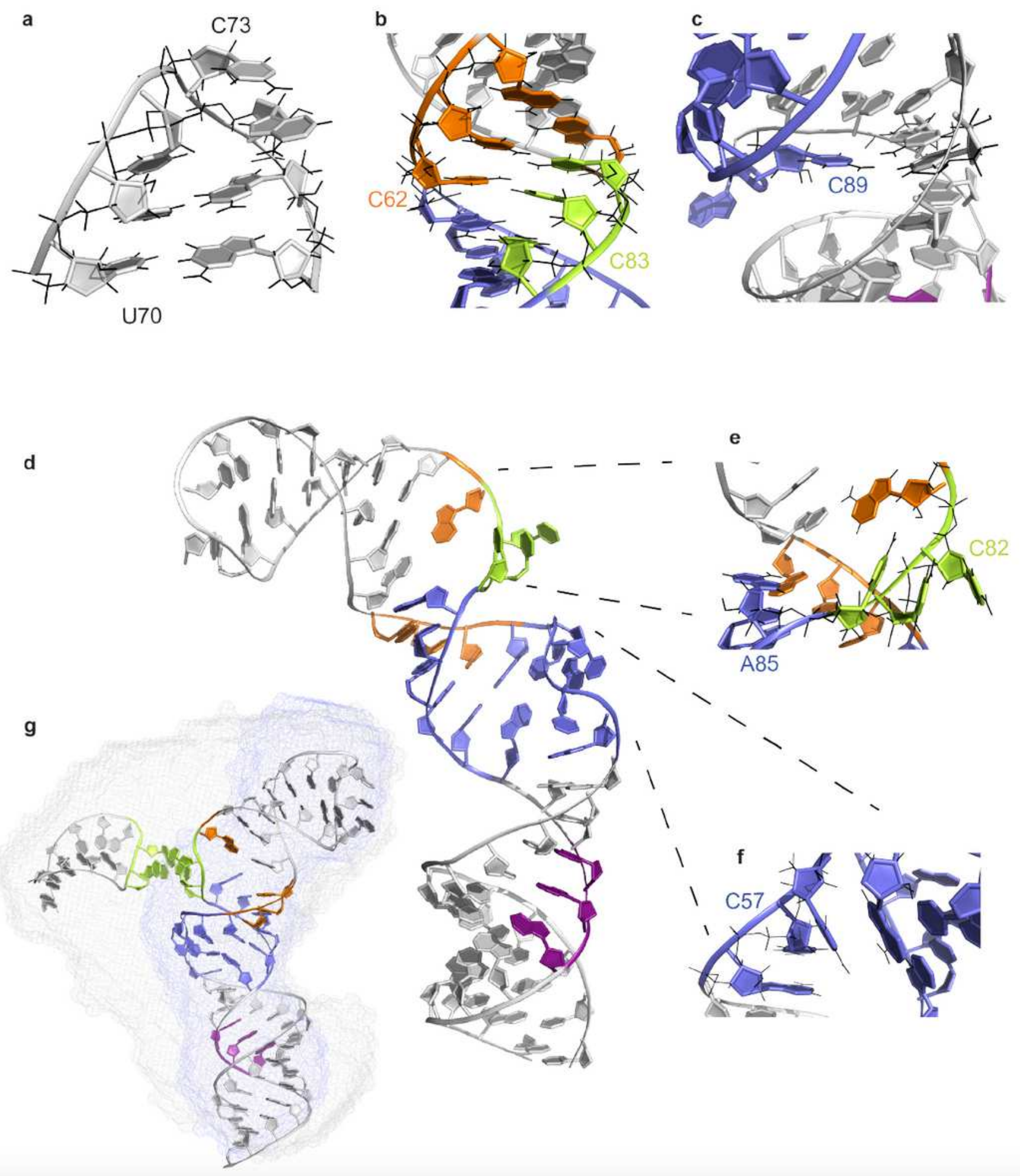

Figure 2

Structural features of SL-Au1. (a) Molecular detail of the C72CA triloop that caps SL-Au1 . (b) Helix 1c of SL-Au1 contains 2 tandem C-C non-canonical basepairs. (c) SL-Au1 is able to form a triple base-pair interaction between C89 and the A92-U54 base-pair. (d) Helix 1c' assumes a kinked conformation and is flanked by an A56CCA bulge at the junction with helix 1b (e) and an A81CCCA bulge at the junction with helix $1 \mathrm{~d}(\mathrm{f})$. (g) The density, as determined by SAXS, of free SL-Au1 in the 1c' conformation (blue) shows 
the bend described above. It also overlays with a model envelope (grey) of the SL-Au1:SL-Ax2 long-range interaction complex. Base coloring is as in Figure 1.
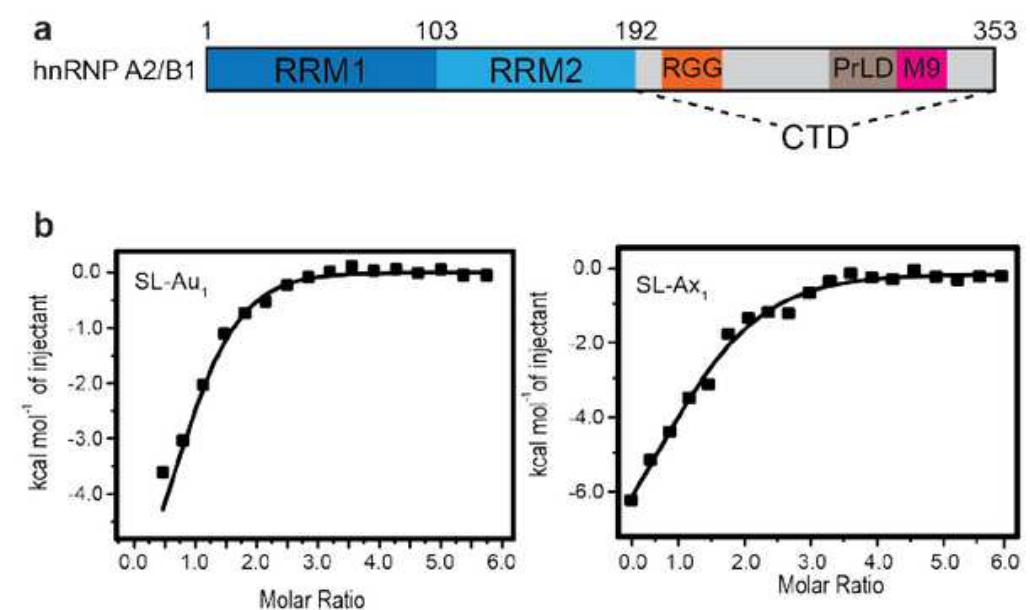

c
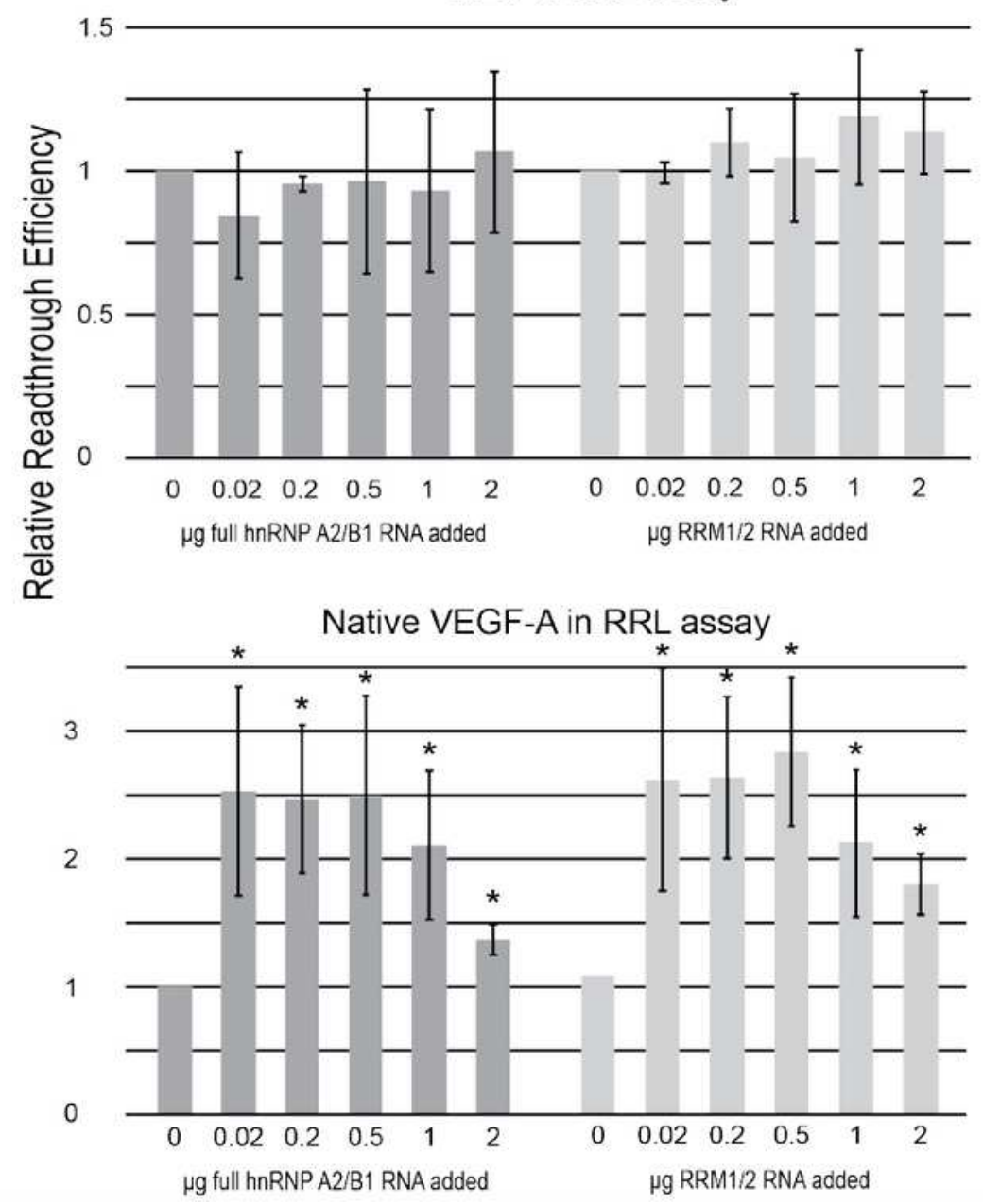

d
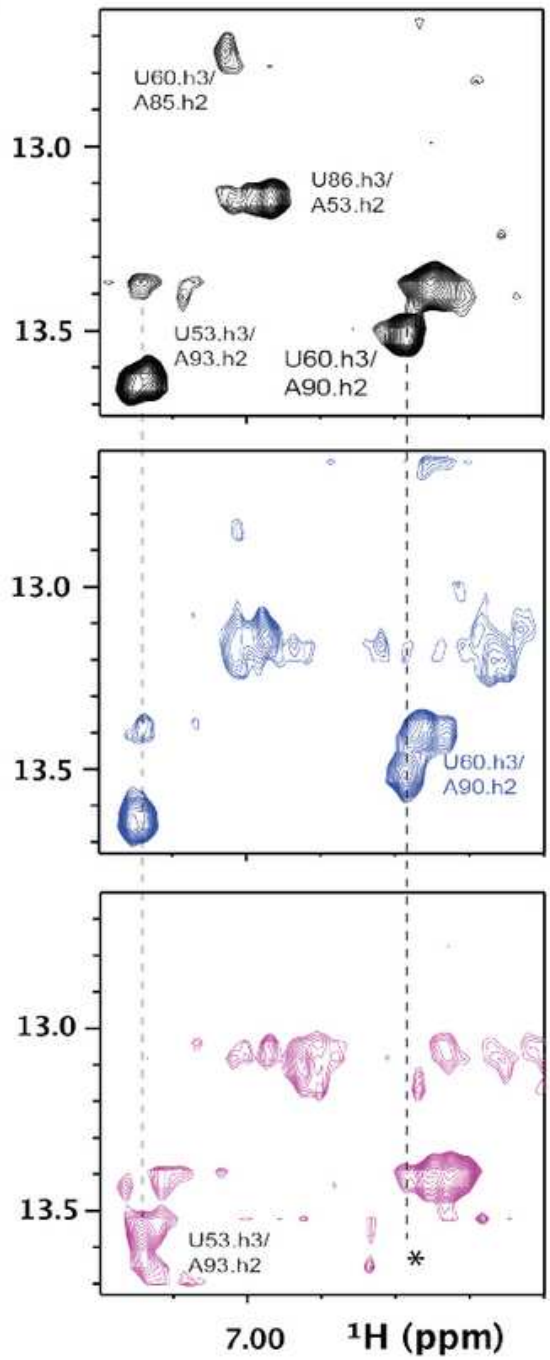

e

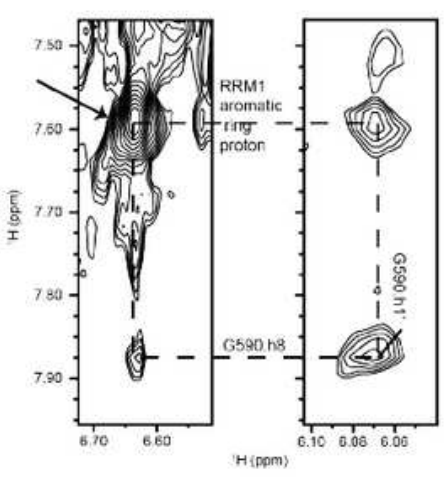

Figure 3

hnRNP A2/B1 binds to VEGF-A mRNA to help mediate readthrough. (a) Schematic of the hnRNP A2/B1 domain architecture showing its RNA-recognition motifs (RRM1 and RRM2) and C terminal domain (CTD), consisting of an Arg-Gly-Gly box (RGG), prion-like domain (PrLD), and M9 transport sequence. (b) 
ITC binding studies of RRM1/2 to SL-Au1 (left) or SL-Ax1 (right) show tight and specific binding. Representative curves of 2 trials are shown. (c) Rabbit reticulocyte lysate assays to which either full length hnRNP A2/B1 (dark grey) mRNA or RRM1/2 (light grey) was added show no difference in readthrough activity for an MLV control construct (top) or a VEGF-A construct (bottom). Error bars indicate standard error $(n=3)$ and statistical tests were performed using a 2-tailed t-test $\left({ }^{*} p<0.05\right)$. (d) Titration of RRM1/2 (black, 0 molar equivalents; blue, 0.3 molar equivalents; pink, 1 molar equivalent) into SL-Au1 at $280 \mathrm{~K}$ shows the shifting of several $1 \mathrm{H}-1 \mathrm{H}$ NMR peaks of base pairs disrupted by protein binding. (e) Titration of RRM1 into SL-Ax 1 at $311 \mathrm{~K}$ results in $1 \mathrm{H}-1 \mathrm{H}$ NOE cross-peaks between $\mathrm{G} 590$ and aromatic ring hydrogens of the protein.
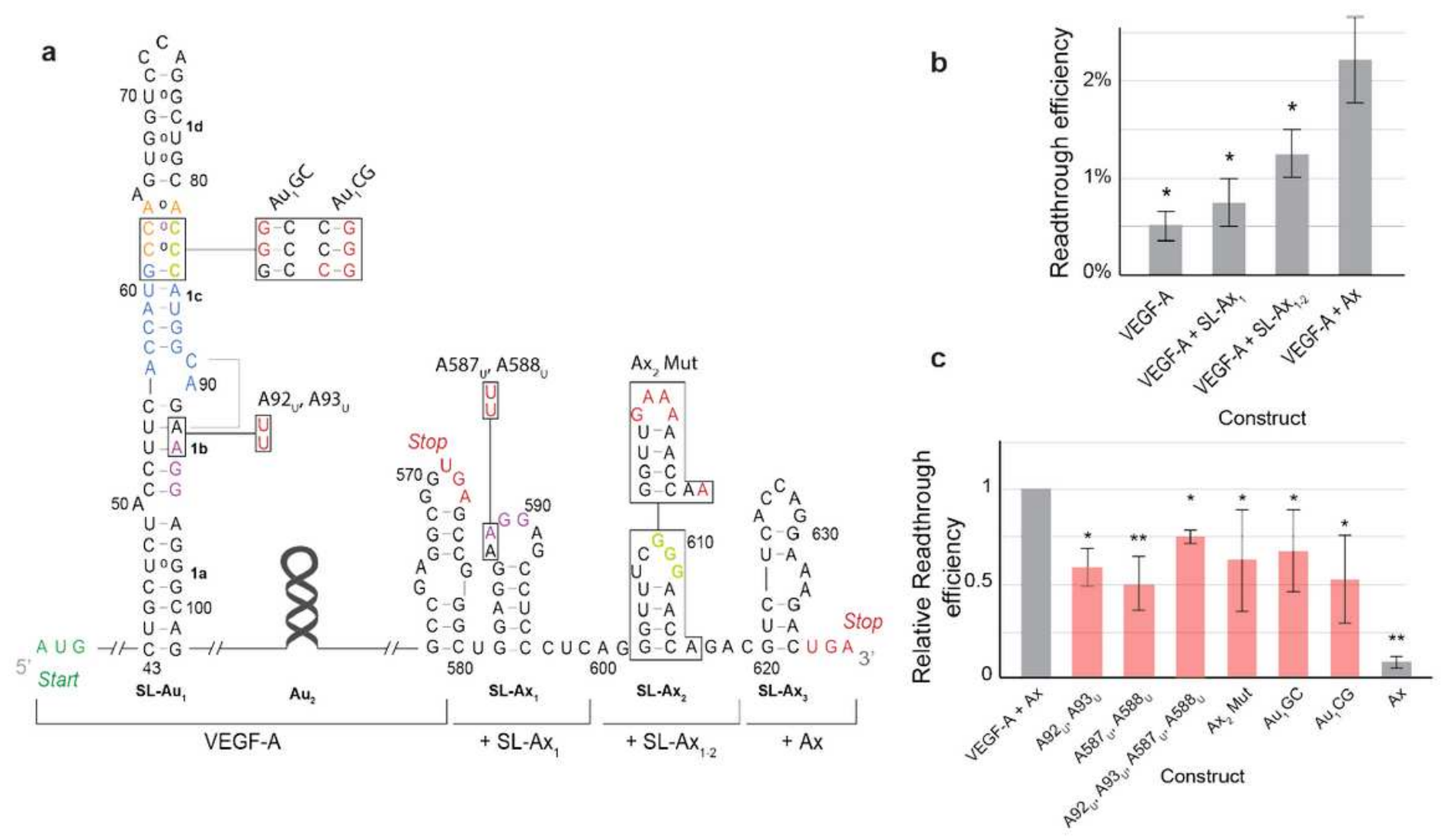

\section{Figure 4}

Each element of the VEGF-mRNA separately contributes toward readthrough activity. (a) Schematic indicating mutations made to the VEGF-A mRNA. Mutations are labeled and shown in red. For truncated products, the maximal extent of the VEGF-A mRNA is indicated underneath the schematic. (b) Functional readthrough experiments show that each stem-loop within the Ax-element contributes toward readthrough. (c) Each tested mutation led to a decrease in readthrough levels, highlighting the importance of each region in mediating ribosomal readthrough. Error bars indicate standard error $(n=3)$ and statistical tests were performed using a 2-tailed t-test $(* p<0.05, * \star p<0.005)$. 


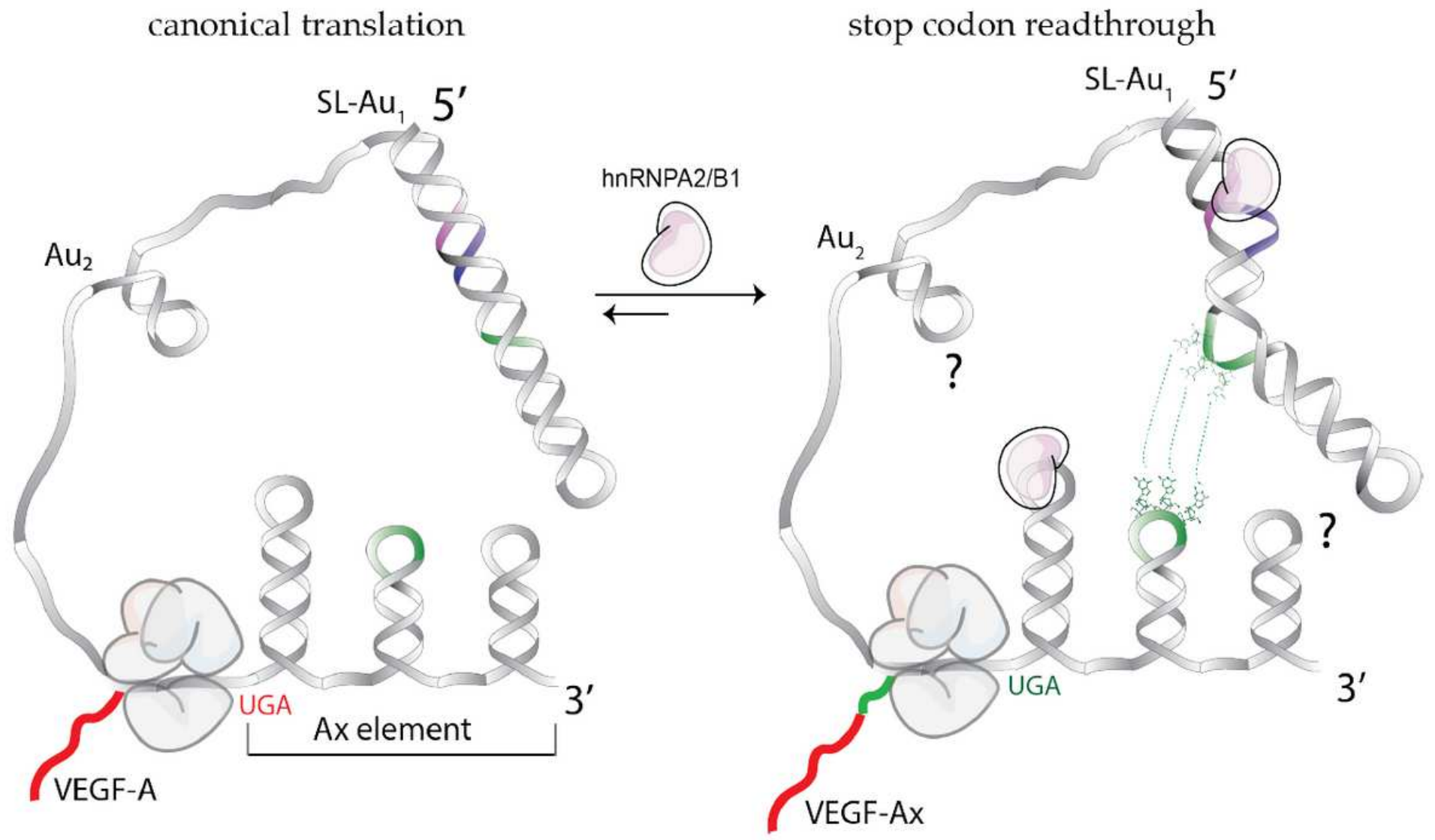

Figure 5

RNA alternate conformations mechanism of VEGF-A readthrough. A mechanistic model of the VEGF-A coding mRNA shows the formation of a linear SL-Au1 element at the $5^{\prime}$ end of the RNA and three stem loops (SL-Ax1-3) at the 3' end of the RNA in the absence of hnRNP A2/B1. In the presence of hnRNP A2/B1, SL-Au1 undergoes a register shift, exposing three cytosine residues. These three cytosine residues are able to partake in long-range Watson-Crick base pairing with SL-Ax2, thereby promoting translational stop-codon readthrough. 